\title{
The Electrospun Ceramic Hollow Nanofibers
}

\author{
Shahin Homaeigohar ${ }^{1, *}$ (D), Yalda Davoudpour ${ }^{2}$, Youssef Habibi ${ }^{3}$ and Mady Elbahri ${ }^{1,4, *}$ \\ 1 Nanochemistry and Nanoengineering, School of Chemical Engineering, Department of Chemistry and \\ Materials Science, Aalto University, Kemistintie 1, 00076 Aalto, Finland \\ 2 The Institute of Mineralogy, Crystallography and Material Science, Faculty of Chemistry and Mineralogy, \\ University of Leipzig, 04109 Leipzig, Germany; y.davoudpour@gmail.com \\ 3 Department of Materials Research and Technology (MRT), Luxembourg Institute of Science and \\ Technology (LIST), L-4362 Esch-sur-Alzette, Luxembourg; youssef.habibi@list.lu \\ 4 Nanochemistry and Nanoengineering, Institute for Materials Science, Faculty of Engineering, \\ Christian-Albrechts-Universität zu Kiel, Kaiserstrasse 2, 24143 Kiel, Germany \\ * Correspondence: shahin.homaeigohar@aalto.fi (S.H.); mady.elbahri@aalto.fi (M.E.); \\ Tel.: +358-50-449-8715 (S.H.); +358-50-431-9831 (M.E.)
}

Received: 28 September 2017; Accepted: 6 November 2017; Published: 9 November 2017

\begin{abstract}
Hollow nanofibers are largely gaining interest from the scientific community for diverse applications in the fields of sensing, energy, health, and environment. The main reasons are: their extensive surface area that increases the possibilities of engineering, their larger accessible active area, their porosity, and their sensitivity. In particular, semiconductor ceramic hollow nanofibers show greater space charge modulation depth, higher electronic transport properties, and shorter ion or electron diffusion length (e.g., for an enhanced charging-discharging rate). In this review, we discuss and introduce the latest developments of ceramic hollow nanofiber materials in terms of synthesis approaches. Particularly, electrospinning derivatives will be highlighted. The electrospun ceramic hollow nanofibers will be reviewed with respect to their most widely studied components, i.e., metal oxides. These nanostructures have been mainly suggested for energy and environmental remediation. Despite the various advantages of such one dimensional (1D) nanostructures, their fabrication strategies need to be improved to increase their practical use. The domain of nanofabrication is still advancing, and its predictable shortcomings and bottlenecks must be identified and addressed. Inconsistency of the hollow nanostructure with regard to their composition and dimensions could be one of such challenges. Moreover, their poor scalability hinders their wide applicability for commercialization and industrial use.
\end{abstract}

Keywords: core-sheath nanofibers; hollow nanofibers; electrospinning; ceramic

\section{Introduction}

Nowadays, one-dimensional (1D) nanostructures, including nanofibers, nanotubes, nanorods, nanowires, and nanobelts have drawn immense attention from various scientific communities. This attraction stems from their unique properties, such as size effects, surface effects, and superparamagnetism, leading to their intriguing applications in many advanced areas including sensors, solar cells, nanoresonators, etc. [1]. Among these 1D nanostructures, nanofibers, with large length/diameter ratios, extensive surface area per unit mass, and small diameters (tens to hundreds of nanometers) have been widely spotlighted. The mentioned structural features bring about flexibility in their applicability.

Up to now, techniques such as drawing, template synthesis, phase separation, self-assembly, and electrospinning have been employed for the fabrication of nanofibers made of polymers, metals, ceramics, glass, etc. These methods not only produce nanofibers, but also further assemble them into two-dimensional (2D) and three-dimensional (3D) nanostructures for practical applications [1].

For the last two decades, an almost old technique, called electrospinning, has been underlined mainly because of its extraordinary capabilities in the production of polymer nanofibers [2-4]. In this regard, 
basic electrospinning theories [5,6] have been thoroughly studied, and a diverse range of electrospun nanofibers from various polymer (synthetic or natural) systems, such as neat, nanohybrid and biohybrid, have been made [7-14]. Electrospinning is, in fact, a novel production technique of continuous ultrafine fibers (with diameters from $10 \mu \mathrm{m}$ to $10 \mathrm{~nm}$ ) based on forcing a polymer melt or solution through a spinneret with an electrical driving force $[15,16]$. The produced mats possess small fiber diameters (as mentioned above), highly specific surface areas (tens to hundreds $\mathrm{m}^{2} / \mathrm{g}$ ), high porosity, and small pore sizes. Thus, they could be excellent candidates for a wide range of applications, including tissue engineering, drug delivery, textiles, filtration, composite reinforcements, etc. [15,17].

The main advantages of electrospinnig are: its relatively easiness (easy to set up), high speed, low cost, high versatility allowing control over the fibers' diameters, microstructures and arrangements, and the possibility of a vast selection of materials $[15,18,19]$. The latter advantage, that is the possibility of fabrication of various nanofiber morphologies and arrangements, is of utmost importance for various applications. By electrospinning, polymers and hybrids thereof can be processed into 1D nanofibers with adjustable compositions, diameters, and porosities [20]. One specific instance is the fabrication of $1 \mathrm{D}$ hollow or tubular nanofibers made of ceramics, and, more specifically, of metal oxides, which have been explored extensively for potential applications in catalysis, chemicals, gas sensors, etc. [21-23].

Hollow nanofibers are able to offer a very extensive surface area that could be highly beneficial for surface-related applications, e.g., chemical sensors, photocatalysis, electromagnetic wave absorbing materials, etc. [24,25]. It is reported that the surface area of the hollow nanofibers is around two times larger than that of the conventional nanofibers [26,27]. In addition to an enhanced surface area, the tubular shape of hollow nanofibers brings about more optimum physicochemical properties for specific applications. For instance, as Choi et al. [28] state, zinc oxide ( $\mathrm{ZnO})$ hollow nanofibers show higher electronic transport properties compared to their thin film counterparts. Also, hollow nanofibers are able to provide promising photocatalytic activity [21,29], increased crystallinity [30] and porosity [31,32], optimized electrochemical [33], magnetic, optical and luminescence properties [34,35], as well as high energy storage capacity [36].

In this review, we aim to present a comprehensive overview of the latest development of ceramic hollow nanofiber materials in terms of synthesis approaches, material types, and applications. The emphasis will be on electrospinning for the production of hollow nanofibers, while, with respect to nanofiber materials, we will consider the most common ceramic (metal oxide) materials that have been particularly studied in relevance with energy and environmental remediation applications.

\section{Electrospinning-Based Fabrication Methods of Ceramic Hollow Nanofibers}

The most widely applied process for the fabrication of hollow nanofibers is electrospinning. Unlike the template synthesis and self-assembly methods relying on complex manipulations of molecules to form the desired structure, electrospinning is an efficient and straightforward method to produce hollow nanofibers from either inorganic or organic components [37].

Electrospinning is an effective, adaptable method to form nanofibers with various structures such as, for example, beads on a string, ribbon, cylindrical, grooved, porous, multichannel, core-shell, side by side, helical, hierarchical, and hollow [38-40]. Electrospun nanofibers are gaining extensive and growing interest because of their flexibility in terms of size, porosity, surface area, morphology, and surface functionality [41,42].

A simple electrospinning apparatus comprises a Direct Current (DC) high voltage source with positive and negative electrodes connected to a spinning solution container with a nozzle (or spinneret) and a collector, respectively. During the process, an electric field is formed between the spinneret and the collector as a result of their opposite charges. Induced by this electric field, a conical shaped droplet, called "Taylor cone", is pulled out from the nozzle. Once the electric force dominates the surface tension of the spinning solution, a charged jet is expelled, the solvent gradually evaporates, and the nanofibers are deposited on the collector [43]. Since 2010, various electrospinning procedures and apparatus have been designed for the fabrication of ceramic hollow nanofibers. These approaches include single nozzle, coaxial, microfluidic, triaxial, and emulsion electrospinning (Table 1). 
Table 1. Various electrospinning methods for the production of ceramic hollow nanofibers, examples, and detailed operational parameters (studies performed after 2010 were mainly considered).

\begin{tabular}{|c|c|c|c|c|}
\hline Method & Precursors & Parameters & Ensuing Hollow Nanofibers & Reference \\
\hline $\begin{array}{l}\text { Single-spinneret } \\
\text { electrospinning }\end{array}$ & $\mathrm{Zn}(\mathrm{AC})_{2} \cdot 2 \mathrm{H}_{2} \mathrm{O}$ in water $/$ Poly(vinylpyrrolidon)(PVP) in DMF & Voltage $=17 \mathrm{kV}$ Distance $=20 \mathrm{~cm}$ & $\mathrm{ZnO}$ & [44] \\
\hline $\begin{array}{l}\text { Coaxial } \\
\text { electrospinning }\end{array}$ & $\begin{array}{l}\mathrm{Fe}_{3} \mathrm{O}_{4} \text { nanoparticles/DMF/Chloroform }\left(\mathrm{CHCl}_{3}\right) / \mathrm{PVP} / \mathrm{Eu}(\mathrm{BA})_{3} \text { phen } \\
\text { powders }\end{array}$ & $\begin{array}{l}\text { Voltage }=11 \mathrm{kV} \text { Distance }=12 \mathrm{~cm} \\
\text { Flow rate }=1 \mathrm{~mL} \cdot \mathrm{h}^{-1}\end{array}$ & $\mathrm{Fe}_{3} \mathrm{O}_{4} / \mathrm{Eu}(\mathrm{BA})_{3}$ phen/PVP & [45] \\
\hline $\begin{array}{l}\text { Coaxial } \\
\text { electrospinning }\end{array}$ & $\begin{array}{l}\mathrm{PVP} / \mathrm{Tb}_{4} \mathrm{O}_{7}, \mathrm{BA}, \mathrm{phen} / \mathrm{FeCl}_{3} \cdot 6 \mathrm{H}_{2} \mathrm{O} / \mathrm{FeSO}_{4} \cdot 7 \mathrm{H}_{2} \mathrm{O} / \mathrm{NH}_{4} \mathrm{NO}_{3}, \\
\text { polyethyleneglycol (PEG)/ammonia/oleic acid (OA)/aniline (ANI), } \\
\text { (IS)-(+)-camphor-10 sulfonic acid (CSA)/ammonium persulfate } \\
\text { (APS)/ethanol }\left(\mathrm{CHCl}_{3}\right) / \mathrm{DMF} / \text { nitric acid/water }\end{array}$ & $\begin{array}{l}\text { Voltage }=13 \mathrm{Kv} \\
\text { Flow rate }=0.0167 \mathrm{~mL} \cdot \mathrm{min}^{-1}\end{array}$ & $\begin{array}{l}\mathrm{Tb}(\mathrm{BA})_{3} \text { phen } / \mathrm{PANI} / \mathrm{Fe}_{3} \mathrm{O}_{4} / \mathrm{PVP} \\
(\mathrm{BA}=\text { benzoic acid, } \\
\text { phen = phenanthroline, } \\
\mathrm{PANI}=\text { polyaniline, } \\
\mathrm{PVP}=\text { polyvinylpyrrolidone })\end{array}$ & [46] \\
\hline $\begin{array}{l}\text { Microfluidic approach } \\
\text { electrospinning }\end{array}$ & $\begin{array}{l}\mathrm{PVP} / \text { tetrabutyl } \\
\text { titanate }\left(\mathrm{Ti}\left(\mathrm{OC}_{4} \mathrm{H}_{9}\right)_{4} / \text { ethanol/paraffin oil }\right.\end{array}$ & $\begin{array}{l}\text { Voltage }=20-30 \mathrm{kV} \\
\text { Distance }=15-25 \mathrm{~cm} \\
\text { Flow rates of inner jet }(\text { paraffin oil })=1.0 \mathrm{~mL} \cdot \mathrm{h}^{-1} \\
\text { Flow rates of outer jet }\left(\mathrm{PVP} / \mathrm{Ti}(\mathrm{OiPr})_{4}\right)=6-12 \mathrm{~mL} \cdot \mathrm{h}^{-1}\end{array}$ & $\mathrm{TiO}_{2}$ & [48] \\
\hline $\begin{array}{l}\text { Triaxial } \\
\text { electrospinning }\end{array}$ & $\begin{array}{l}\text { tetraethyl orthosilicate (TEOS)/ethanol/water/HCl (shell and } \\
\text { innermost layers)+poly(styrene-b-isoprene)(middle layer) }\end{array}$ & $\begin{array}{l}\text { Voltage }=20 \mathrm{kV} \\
\text { Distance }=11.5 \mathrm{~cm} \\
\text { Flow rates of inner jet }=0.02 \mathrm{~mL} \cdot \mathrm{min}^{-1} \\
\text { Flow rates of outer jet }=0.03 \mathrm{~mL} \cdot \mathrm{min}^{-1}\end{array}$ & $\mathrm{SiO}_{2} / \mathrm{PS}-\mathrm{b}-\mathrm{PI} / \mathrm{SiO}_{2}$ & [49] \\
\hline $\begin{array}{l}\text { Emulsion } \\
\text { electrospinning }\end{array}$ & $\mathrm{PVP} / \mathrm{VO}(\mathrm{acac})_{2} / \mathrm{HAuCl}_{4} \cdot 3 \mathrm{H}_{2} \mathrm{O}-\mathrm{DMF}$ solution and PS-DMF solution & $\begin{array}{l}\text { Voltage }=5-30 \mathrm{kV} \text { Distance }=7 \mathrm{~cm} \\
\text { Flow rate }=2 \mathrm{~mL} \cdot \mathrm{h}^{-1}\end{array}$ & $\mathrm{Au} / \mathrm{V}_{2} \mathrm{O}_{5}$ & {$[50]$} \\
\hline
\end{tabular}




\subsection{Electrospinning with a Single Spinneret}

The single-spinneret, or single-nozzle, electrospinning is the simplest electrospinning procedure to form hollow nanofibers from either one component or multiple components [44,52-55]. The schematic of this process is illustrated in Figure 1. In the case of multicomponents electrospinning, polymers with high and low viscosity move to the inner and outer layers, respectively [56]. The rheological parameters, the solubility of the constituents, and the phase separation of the employed blends influence the homogeneity of their respective solutions [57]. The main challenge in this technique is its low throughput, varying between 1 and $5 \mathrm{~mL} / \mathrm{h}$ depending on the flow rate of 0.1 to $1 \mathrm{~g} / \mathrm{h}$ (that is based on the fiber weight), and depending on the operating factors as well as on the solution properties [58,59]. Generally, decreasing the amount of solid materials in the spinning solution as well as the flow rate, declines the nanofibers diameter and the throughput [60]. Despite the simplicity of this process in the lab scale, a high production rate is necessary for industrial and commercial purposes. Moreover, there is a limited number of common solvents to prepare a blend polymer solution, and it is also challenging to find optimum electrospinning conditions for different polymers in a blend solution $[20,61]$.

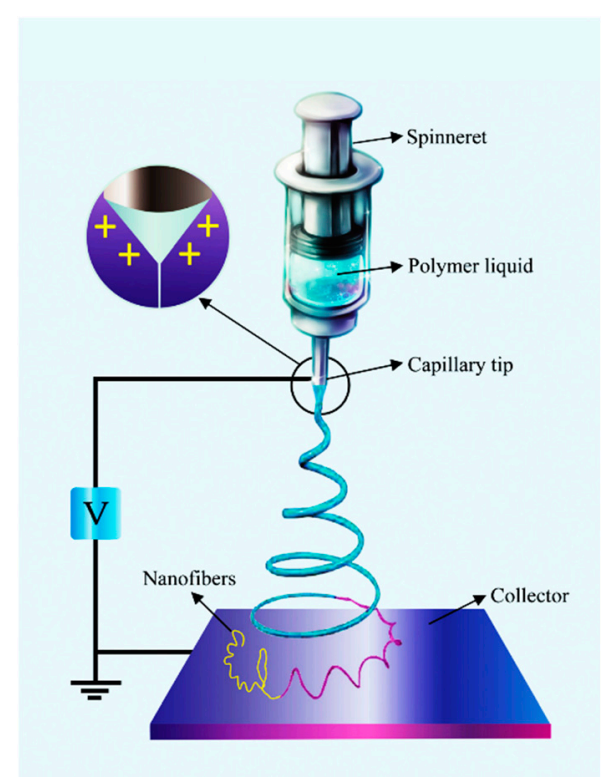

Figure 1. A schematic of the single-spinneret electrospinning (Reproduced with permission from [62]. Royal Society of Chemistry, 2017).

\subsection{Coaxial Electrospinning with a Two-Capillary Spinneret}

Among the derivative techniques of electrospinning, coaxial electrospinning is indeed the most widely employed for the production of hollow nanofibers of polymers $[46,63,64]$, ceramics $[47,65]$, metals [26,45,47,66], and carbon [67-69]. In this technique, two different solutions are first fed into a spinneret comprised of two coaxial capillaries, to form a core (inner layer)-sheath (outer layer)-nanofiber structure. Subsequently, by removal of the core via calcination, solvent extraction, or washing, hollow nanofibers are produced $[26,46,69,70]$. Figure 2a shows schematically the preparation process of core-sheath $\mathrm{TiO}_{2}$ nanofibers using a coaxial electrospinning set-up [47]. By removal of the core, hollow $\mathrm{TiO}_{2}$ nanofibers are made. Scanning Electron Microscopy (SEM) images of the produced core-sheath, then $\mathrm{TiO}_{2}$ hollow nanofibers are shown in Figure $2 \mathrm{~b}-\mathrm{d}$. 

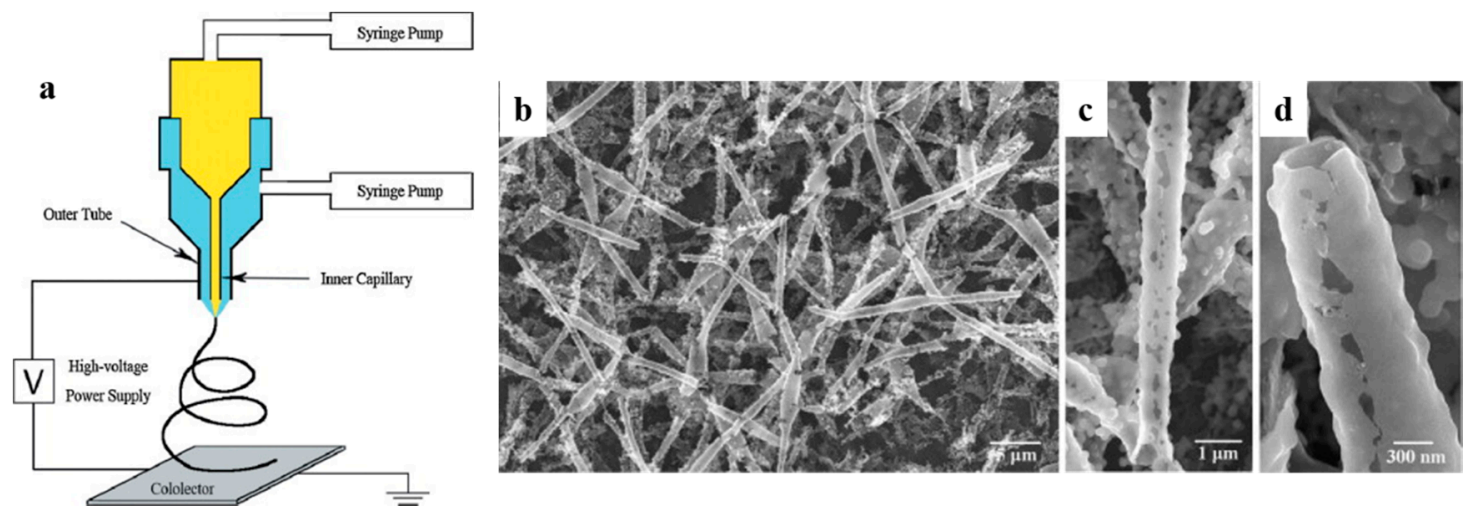

Figure 2. (a) A schematic of the coaxial electrospinning process for the fabrication of core-sheath $\mathrm{TiO}_{2}$ nanofibers; SEM images of the core-sheath, then hollow $\mathrm{TiO}_{2}$ nanofibers $\left(\mathrm{TiO}_{2} / \mathrm{PVP}\right.$ composite nanofibers were coaxially electrospun with a PVP core solution and a titanium precursor as the shell solution, then calcined at $550{ }^{\circ} \mathrm{C}$ for $3 \mathrm{~h}$ ); (b) a low magnification image of the as-synthesized hollow $\mathrm{TiO}_{2}$ nanofibrous mat; (c) a high magnification image of the $\mathrm{TiO}_{2}$ nanofibers; and (d) a high magnification image of the cross section of the hollow $\mathrm{TiO}_{2}$ nanofiber (Reproduced with permission from [47]. Elsevier, 2017).

Since the sheath and core solutions meet at the end of the nozzle, two physical phenomena take place simultaneously: the wrapping of the sheath solution around the core solution and the formation of the Taylor cone by the sheath solution to equilibrate the charge effect and the fluid surface tension [71]. Hence, for a successful electrospinning, two important relevant issues should be taken into account. Firstly, the core and the sheath solutions should be immiscible [70,72]. The incompatibility between the core and the sheath solutions governs a gelled interface wherein, by coagulation of both solutions, the hollow nanofibers are produced [73]. Secondly, the sheath solution should be spinnable to impose a shear stress on the core solution while pulling the blended droplet. The core solution can be spinnable or not $[47,74]$. The flow and diffusion rates, the viscosity, and the miscibility are critical parameters in the coaxial electrospinning process [56]. In contrast to the single-nozzle electrospinning, the key advantage of this method is the possibility of fabrication of hollow nanofibers from a wide variety of materials, even non-electrospinnable solutions [69]. However, Wei et al. [75] have reported the following limitations for this approach:

- The sheath layer must be strong enough to retain the hollow structure, otherwise the produced hollow nanofibers will collapse.

- Despite the easiness of this method, continuous and perfect hollow nanofibers are hardly made because of the post-treatment processes applied to remove the core.

- Complete elimination of the core is challenging.

- The hollow nanofibers prepared by this method can consist of only one layer wall.

Additionally, the limited number of suitable inner solvents and the lack of control over the electrospinning parameters are other problems that can hinder the applicability of the coaxial electrospinning for some systems [76].

\subsection{Microfluidic Electrospinning}

If the inner needle of the coaxial electrospinning possesses two or more channels, multichannel hollow nanofibers can be made $[48,77,78]$. In this procedure, called microfluidic or multifluidic coaxial electrospinning, the apparatus consists of several inner capillaries with an outer nozzle. The outer and inner solutions are separately fed into the capillaries and form a compound Taylor cone that is stretched under an applied electric field and solidified to multichannel nanofibers [48]. Figure 3a illustrates the schematic of a microfluidic electrospinning set-up that is able to fabricate a nanowire-in-microtube 
structure. This interesting structure is visualized by SEM and Transmission Electron Microscopy (TEM) images (Figure 3b). Compared to the traditional coaxial electrospinning, the microfluidic approach reduces the interaction of the sheath and core fluids, which could be highly miscible or undergo rapid phase separation, by introducing an extra middle fluid as a separator. Thus, a wider range of fluid pairs can be regarded [77]. In addition, the form, size and composition of nanofibers can be properly controlled, as it is desired in the textile and biomedical fields [79]. Other advantages of this process are: simplicity, controllable channel size, rapid prototyping, and parallel spinnability of multiple fibers via arrays of single microchannels [80-82]. However, since the inner fluid is surrounded by the middle one, the evaporation of the respective solvents during the process is limited. Thus, this method suffers from a difficulty in the solvent recovery [82].

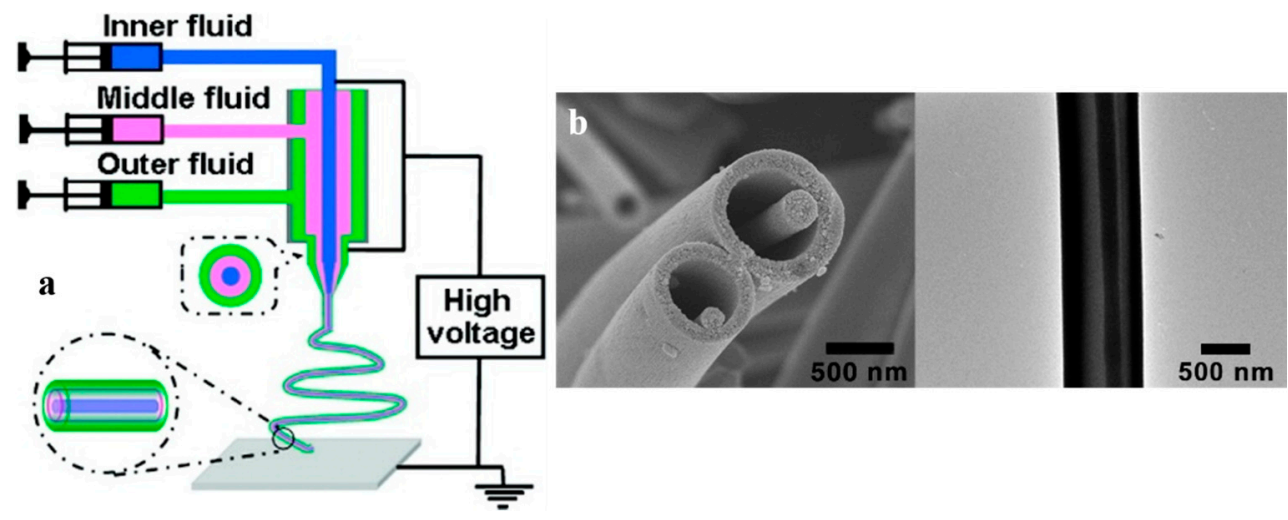

Figure 3. (a) The schematic shows the configuration of the microfluidic electrospinning set-up employed to produce hollow $\mathrm{TiO}_{2}$ fibers with a nanowire-in-microtube structure. The main spinneret consists of three coaxial capillaries, whereby three fluids are fed to form a compound jet when a high electric field is applied. Among the fluids, the middle one acts as a spacer and separates the inner and outer fluids. (b) SEM (left) and TEM (right) images represent the developed nanowire-in-microtube structure (Reproduced with permission from [77]. American Chemical Society, 2017).

\subsection{Triaxial Electrospinning}

Triaxial electrospinning employs a spinneret with three concentric needles (Figure 4a). As seen in Figure $4 b$, three different solutions are pumped into and then meet at the tip of the spinneret. Similar to other electrospinning approaches, the compound solution deforms into a Taylor cone under an electrostatic field. The surface tension of the solution dominates upon the electrostatic force and, thus, a triaxial jet emerges that then experiences bending instability, whipping motion, and solvent evaporation, and eventually it is deposited on the collector as dry fibers [83,84]. This procedure produces three-layered nanofibers including inner (core), intermediate, and outer (sheath) layers. The intermediate layer acts as a barrier between the sheath and the core regions [85].

To successfully perform the triaxial electrospinning, a compound Taylor cone must be formed, and the three involved fluids should be held concentrically together during the procedure [84]. Moreover, it is crucial to select an appropriate solvent for each component, with a boiling point that prevents the solvent from rapidly evaporating, which would damage the structure of the formed nanofibers [86]. The boiling point of the outer layer should be lower than that of the inner layer. Also, the molecular weight of the inner layer should be comparable, or even lower than that of the outer layer.

Triaxial electrospinning has been applied to fabricate hollow nanofibers from a wide variety of materials [87,88]. For instance, Joo et al. [49] fabricated triaxial electrospun fibers with silica as the shell and core layers, and with a self-assembling polymeric material as the intermediate layer. Also, Chen et al. [77] produced nanowire-in-microtube structured nanofibers through triaxial electrospinning. 

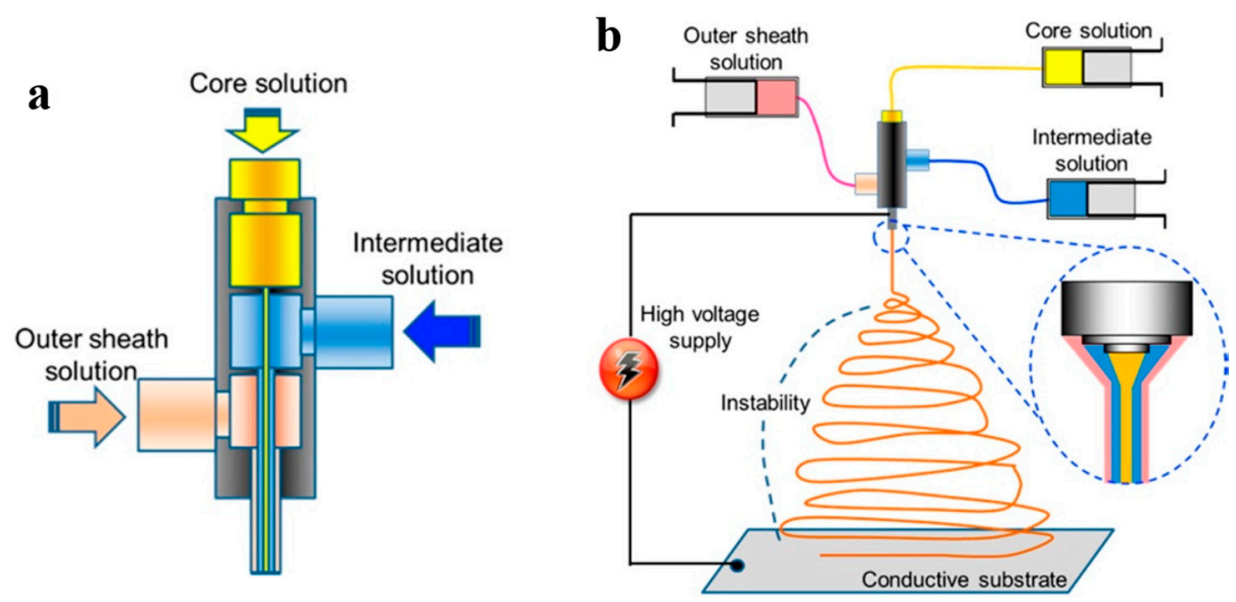

Figure 4. Triaxial electrospinning process: (a) triaxial spinneret; (b) basic mechanism (Reproduced with permission from [85]. American Chemical Society, 2017).

The main advantage of this technique is the possibility of formation of nanofibers with a higher surface area enabling the sustained release of important agents [86]. For instance, multidrug delivery vehicles with various release times that are able to sustainably release drugs and improve the healing process can be produced by triaxial electrospinning [85]. Moreover, triaxial electrospinning enables the production of nanofibers from non-electrospinnable components. However, problems such as needle blocking and configuration complexity of this system are challenging $[59,89,90]$.

\subsection{Emulsion Electrospinning}

Emulsion electrospinning, which is the electrospinning of a blend of two immiscible liquid phases, is principally similar to solution electrospinning, but different in chemistry [91,92]. Through this method, discontinuous core-sheath nanofibers are formed by stretching and collapsing of an emulsion $[57,93]$. Chemically, two dissimilar polymers are dissolved in a solvent, mixed, and settled to produce an emulsion in which the core and sheath segments form from dispersed drops and the continuous phase, respectively $[56,91,94,95]$. To maintain the stability of the emulsion before and during the jet formation, an emulsifier is usually used. In addition to the stability of the emulsion, the viscosity of the drop phase should be optimum for deformation [56,94,95].

There are two types of emulsion for the electrospinning process: water in oil $(\mathrm{W} / \mathrm{O})$ and oil in water $(\mathrm{O} / \mathrm{W})[95,96]$. In a $\mathrm{W} / \mathrm{O}$ system, the viscosity of the water phase is lower than that of the oily one. Hence, the tendency of the oily phase to form the sheath as a result of its higher viscosity is larger $[56,94]$. For an $\mathrm{O} / \mathrm{W}$ system, the situation is the opposite.

Emulsion electrospinning does not require complex spinnerets compared to the coaxial electrospinning, and it could simply provide good concentric core-sheath nanofibers [97,98]. Another advantage of this method is its eco-friendliness since it employs water rather than organic solvents, and, because of its large dielectric constant, small nanofibers form quickly [95,99]. However, this method suffers from some difficulties in the preparation of a proper emulsion, the elimination of the core, the removal of the emulsifier, which may raise biocompatibility concerns, and a low continuity of the formed hollow nanofibers [52,93].

Among the studies on the preparation of core-sheath nanofibers through emulsion electrospinning, Wang et al. [51] made $\mathrm{TiO}_{2}$ nanotubes via emulsion electrospinning of a $\mathrm{W} / \mathrm{O}$ system. As shown in Figure $5 \mathrm{a}(\mathrm{I})$, they electrospun a homogenous solution containing $\mathrm{PVP}$ and a $\mathrm{TiO}_{2}$ precursor (tetrabutyl titanate), wherein a mechanical pump oil was dispersed. During the electrospinning, as shown in Figure 5a (II), the solvent immediately evaporated, leading to the formation of PVP/tetrabutyl titanate nanofibers. The nanofibers also contained oil drops that were insoluble in the precursor solution. As seen in Figure 5a (III), upon drying the electrospun nanofibers at $60^{\circ} \mathrm{C}$ for $6 \mathrm{~h}$, the dispersed 
microdrops of oils coalesce and form larger oil phases. Eventually, when the nanofibers are annealed at $500{ }^{\circ} \mathrm{C}$ for $2 \mathrm{~h}$, the oil readily evaporates, and $\mathrm{TiO}_{2}$ nanotubes are created (Figure $5 \mathrm{a}(\mathrm{IV})$ ).
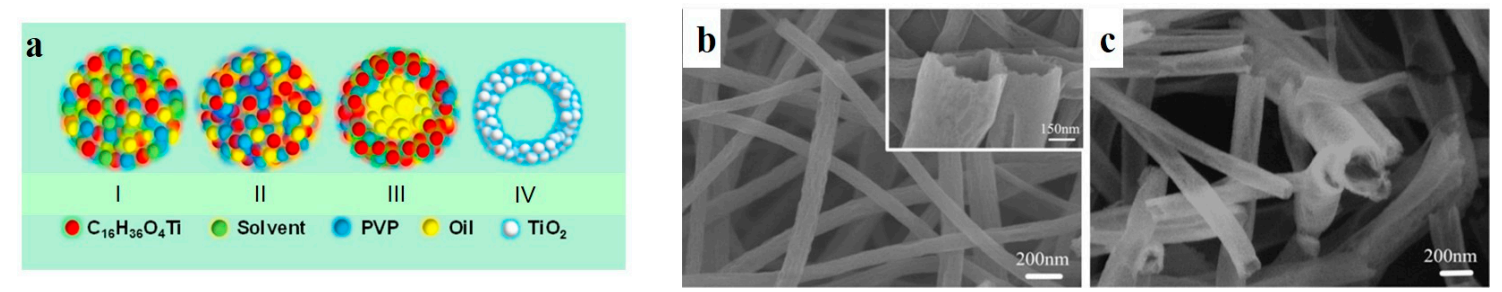

Figure 5. (a) The schematic illustrates the formation mechanism of $\mathrm{TiO}_{2}$ nanotubes by emulsion electrospinning; SEM images of $(\mathbf{b})$ neat $\mathrm{TiO}_{2}$ nanotubes (the inset image verifies the nanotubular morphology and rough surface of the formed nanotubes) and (c) $\mathrm{Ag} / \mathrm{TiO}_{2}$ nanotubes (1.5\%) (Reproduced with permission from [51]. Elsevier, 2017).

\section{Most Studied Ceramic Hollow Nanofibers and Their Applications}

With respect to hollow nanofiber materials, ceramic - particularly metal oxide-hollow nanofibers have attracted a wide research interest because of their special morphologies, compositions, and chemical and physical properties (e.g., adsorptivity, conductivity etc.) [100]. More specifically, they confer unique electrical, electrochemical, and catalytic properties that are associated with their high surface/volume ratio. Also, in some instances, they offer extraordinary transport properties induced by confinement effects, 1D transport phenomena, or the transport in fractal dimensions [28]. These unique, optimized properties have motivated researchers to employ metal oxide hollow nanofibers as ideal building blocks for a wide range of applications. For instance, they have been evaluated as conductive electrodes for optoelectronic devices (e.g., solar cells [101]), dye adsorbents [102], gas sensors [103,104], chemical sensors [28], etc. In the following table (Table 2), a list of the metal oxide hollow nanofibers developed since 2010 is presented. Afterwards, the most well-known examples of metal oxide hollow nanofibers with their respective applications will be introduced. 
Table 2. Various metal oxide hollow nanofibers and their detailed electrospinning parameters.

\begin{tabular}{|c|c|c|c|c|}
\hline Hollow Nanofiber & Precursors & $\begin{array}{l}\text { Electrospinning } \\
\text { Conditions }\end{array}$ & Annealing Conditions & Reference \\
\hline \multirow{3}{*}{$\mathrm{TiO}_{2}$} & PVP/Tetra-butyl titanate (TBT)/ethanol/acetic acid & $\begin{array}{l}V=30 \mathrm{kV} \\
D=15 \mathrm{~cm}\end{array}$ & $\begin{array}{c}T=500^{\circ} \mathrm{C} \\
t=4 \mathrm{~h} \\
\mathrm{HR}=2^{\circ} \mathrm{C} \cdot \min ^{-1}\end{array}$ & [105] \\
\hline & $\mathrm{PVP} /$ tetrabutyl titanate $\left(\mathrm{Ti}\left(\mathrm{OC}_{4} \mathrm{H}_{9}\right)_{4}\right) /$ ethanol/paraffin oil & $\begin{array}{c}V=20-30 \mathrm{kV} \\
D=15-25 \mathrm{~cm} \\
\text { FR (outer) }=6-12 \mathrm{~mL} \cdot \mathrm{h}^{-1} \\
\text { FR (inner) }=1 \mathrm{~mL} \cdot \mathrm{h}^{-1}\end{array}$ & $\begin{array}{c}T=500^{\circ} \mathrm{C} \\
t=8 \mathrm{~h}\end{array}$ & [48] \\
\hline & Titanium isopropoxide/PVP/acetic acid/ethanol & $\begin{array}{c}V=30 \mathrm{kV} \\
D=20 \mathrm{~cm} \\
\mathrm{FR}=0.1 \mathrm{~mL} \cdot \mathrm{h}^{-1}\end{array}$ & $\begin{array}{c}T=600{ }^{\circ} \mathrm{C} \\
t=2 \mathrm{~h}\end{array}$ & [66] \\
\hline $\mathrm{SnO}_{2} / \mathrm{TiO}_{2}$ & Titanium isopropoxide/PVP/acetic acid/ethanol & $\begin{array}{c}V=5 \mathrm{kV} \\
\text { FR (outer) }=1 \mathrm{~mL} \cdot \mathrm{h}^{-1} \\
\text { FR (inner) }=0.1 \mathrm{~mL} \cdot \mathrm{h}^{-1}\end{array}$ & $\begin{array}{c}T=500{ }^{\circ} \mathrm{C} \\
t=2 \mathrm{~h}\end{array}$ & [106] \\
\hline \multirow{4}{*}{$\mathrm{TiO}_{2}$} & $\begin{array}{l}\text { Titaniumisopropoxide(TiP)/poly (methylmethacrylate)(PMMA)/hexadecyl } \\
\text { trimethylammoniumbromide/paraffin oil/methylene chloride/ethanol/acetic acid }\end{array}$ & $\begin{array}{c}V=18 \mathrm{kV} \\
D=15 \mathrm{~cm} \\
\mathrm{FR}=100 \mu \mathrm{L} \cdot \mathrm{min}^{-1}\end{array}$ & $T=500{ }^{\circ} \mathrm{C}$ & [97] \\
\hline & Titanium butoxide (TBT, $\left.\mathrm{I}(\mathrm{OBu})_{4}\right) / \mathrm{PVP} /$ ethylene glycol (EG)/ethanol/acetic acid & $\begin{array}{c}V=0-50 \mathrm{kV} \\
D=50 \mathrm{~cm} \\
\text { FR }=100 \mu \mathrm{L} \cdot \mathrm{min}^{-1}\end{array}$ & $\begin{array}{c}T=550^{\circ} \mathrm{C} \\
t=3 \mathrm{~h} \\
\mathrm{HR}=2^{\circ} \mathrm{C} \cdot \min ^{-1}\end{array}$ & [47] \\
\hline & Titanium (IV) N-butoxide (TNBT)/PVP/ethanol/paraffin oil & $\begin{aligned} V & =15 \mathrm{kV} \\
\text { FR (outer) } & =0.8 \mathrm{~mL} \cdot \mathrm{h}^{-1} \\
\text { FR (inner) } & =0.6 \mathrm{~mL} \cdot \mathrm{h}^{-1}\end{aligned}$ & $\begin{array}{c}T=500{ }^{\circ} \mathrm{C} \\
t=6 \mathrm{~h}\end{array}$ & [107] \\
\hline & $\begin{array}{l}\text { polyacrylonitrile (PAN)/PVP/dimethylformamide (DMF)/tetrabutyl titanate } \\
\left(\mathrm{Ti}_{(}\left(\mathrm{OC}_{4} \mathrm{H}_{9}\right)_{4}\right)\end{array}$ & & $\begin{aligned} T & =500{ }^{\circ} \mathrm{C} \\
t & =5 \mathrm{~h}\end{aligned}$ & [108] \\
\hline $\mathrm{BaTiO}_{3}$ & Barium acetate/acetic acid/Titanium (IV)-isopropoxide/PVP/ethanol & $\begin{array}{c}V=12 \mathrm{kV} \\
D=15 \mathrm{~cm} \\
\mathrm{FR}=0.3 \mu \mathrm{L} \cdot \mathrm{s}^{-1}\end{array}$ & $\begin{array}{c}\mathrm{T}=500,700,950^{\circ} \mathrm{C} \\
t=1 \mathrm{~h} \\
\mathrm{HR}=2.5^{\circ} \mathrm{C} \cdot \mathrm{min}^{-1}\end{array}$ & [109] \\
\hline Carbon nanotube $(\mathrm{CNT})-\mathrm{TiO}_{2}$ & $\begin{array}{l}\text { PAN/Multiwalled CNTs (MWCNTs)/DMF/titanium tetra-isopropoxide } \\
\text { (TTIP)/isopropyl alcohol }\end{array}$ & $\begin{array}{c}V=18 \mathrm{kV} \\
D=10 \mathrm{~cm} \\
\mathrm{FR}=1 \mathrm{~mL} \cdot \mathrm{h}^{-1}\end{array}$ & $\begin{aligned} T & =550^{\circ} \mathrm{C} \\
t & =1 \mathrm{~h}\end{aligned}$ & [110] \\
\hline
\end{tabular}


Table 2. Cont

\begin{tabular}{|c|c|c|c|c|}
\hline Hollow Nanofiber & Precursors & $\begin{array}{l}\text { Electrospinning } \\
\text { Conditions }\end{array}$ & Annealing Conditions & Reference \\
\hline \multirow[b]{2}{*}{$\mathrm{TiO}_{2}$} & $\begin{array}{l}\text { Butyl titanate (TBOT)/diiso-propyl azodiformate (DIPA)/paraffin oil/ethyl alcohol/acetic } \\
\text { acid/deionized water }\end{array}$ & $\begin{aligned} V & =18 \mathrm{kV} \\
D & =20 \mathrm{~cm} \\
\mathrm{FR} & =1 \mathrm{~mL} \cdot \mathrm{h}^{-1}\end{aligned}$ & $\begin{aligned} T & =500^{\circ} \mathrm{C} \\
t & =3 \mathrm{~h} \\
\mathrm{HR} & =1^{\circ} \mathrm{C} \cdot \min ^{-1}\end{aligned}$ & [55] \\
\hline & $\begin{array}{l}\text { Polyvinyl acetate (PVAc)/titanium isopropoxide (TIP)/DMF/calcium carbonate } \\
\qquad\left(\mathrm{CaCO}_{3}\right) / \text { hydrochloric acid }(\mathrm{HCl})\end{array}$ & $\begin{aligned} V & =17 \mathrm{kV} \\
D & =18 \mathrm{~cm} \\
\mathrm{FR} & =1 \mathrm{~mL} \cdot \mathrm{h}^{-1}\end{aligned}$ & $\begin{array}{l}T=500{ }^{\circ} \mathrm{C} \\
t=3 \mathrm{~h}\end{array}$ & [111] \\
\hline $\mathrm{Pt} / \mathrm{TiO}_{2}$ & $\begin{array}{l}\text { Tetrabutyl titanate }\left(\mathrm{Ti}\left(\mathrm{OC}_{4} \mathrm{H}_{9}\right)_{4}, \mathrm{TBOT}\right) / \text { ethanol/hexachloro-platinic acid } \\
\qquad\left(\mathrm{H}_{2} \mathrm{PtCl}_{6} \cdot 6 \mathrm{H}_{2} \mathrm{O}\right) / \mathrm{PVP} / \mathrm{Nitric} \text { acid }\left(\mathrm{HNO}_{3}\right)\end{array}$ & $\begin{aligned} V & =25 \mathrm{kV} \\
D & =25 \mathrm{~cm} \\
\mathrm{FR}=1.3 & \pm 0.02 \mathrm{~mL} \cdot \mathrm{h}^{-1}\end{aligned}$ & $\begin{aligned} T= & 350-500{ }^{\circ} \mathrm{C} \\
& t=4 \mathrm{~h}\end{aligned}$ & [112] \\
\hline $\mathrm{CoFe}_{2} \mathrm{O}_{4}$ & $\mathrm{PVP} / \mathrm{Fe}\left(\mathrm{NO}_{3}\right)_{3} \cdot 9 \mathrm{H}_{2} \mathrm{O} / \mathrm{Co}\left(\mathrm{NO}_{3}\right)_{2} \cdot 6 \mathrm{H}_{2} \mathrm{O} / \mathrm{ethanol} /$ water & $\begin{aligned} V & =30 \mathrm{kV} \\
D & =15 \mathrm{~cm} \\
\mathrm{FR}=1.3 & \pm 0.02 \mathrm{~mL} \cdot \mathrm{h}^{-1}\end{aligned}$ & $\begin{array}{c}T=500-600-700^{\circ} \mathrm{C} \\
\quad t=4 \mathrm{~h} \\
\mathrm{HR}=3^{\circ} \mathrm{C} \cdot \min ^{-1}\end{array}$ & [113] \\
\hline $\mathrm{CuFe}_{2} \mathrm{O}_{4}$ & $\mathrm{PVP} / \mathrm{Fe}\left(\mathrm{NO}_{3}\right)_{3} \cdot 9 \mathrm{H}_{2} \mathrm{O} / \mathrm{Cu}\left(\mathrm{NO}_{3}\right)_{2} \cdot 3 \mathrm{H}_{2} \mathrm{O} /$ ethanol/water & $\begin{array}{l}V=15 \mathrm{kV} \\
D=15 \mathrm{~cm}\end{array}$ & $\begin{aligned} T & =500{ }^{\circ} \mathrm{C} \\
\quad t & =2 \mathrm{~h} \\
\mathrm{HR} & =0.5^{\circ} \mathrm{C} \cdot \mathrm{min}^{-1}\end{aligned}$ & [114] \\
\hline $\mathrm{CoFe}_{2} \mathrm{O}_{4}-\mathrm{PANI}$ & $\begin{array}{l}\text { Cobalt(II) nitrate hexahydrate }\left(\mathrm{Co}\left(\mathrm{NO}_{3}\right)_{2} \cdot 6 \mathrm{H}_{2} \mathrm{O} / \text { iron(III) nitrate enneahydrate }\right. \\
\left(\mathrm{Fe}\left(\mathrm{NO}_{3}\right)_{3} / \text { ethanol/PVP/ammonium peroxodisulfate }\right.\end{array}$ & $\begin{aligned} V & =20 \mathrm{kV} \\
D & =17 \mathrm{~cm} \\
\mathrm{FR} & =0.5 \mathrm{~mL} \cdot \mathrm{h}^{-1}\end{aligned}$ & $\begin{array}{c}T=550^{\circ} \mathrm{C} \\
\quad t=2 \mathrm{~h} \\
\mathrm{HR}=5^{\circ} \mathrm{C} \cdot \min ^{-1}\end{array}$ & [115] \\
\hline $\mathrm{SrFe}_{12} \mathrm{O}_{19}$ & $\begin{array}{l}\text { Strontium nitrate }\left(\mathrm{Sr}\left(\mathrm{NO}_{3}\right)_{2}\right) / \text { Ferric nitrate } \\
\qquad\left(\mathrm{Fe}\left(\mathrm{NO}_{3}\right)_{3} \cdot 9 \mathrm{H}_{2} \mathrm{O}\right) / \mathrm{PVP} / \mathrm{DMF}\end{array}$ & $\begin{aligned} V & =15 \mathrm{kV} \\
D & =15 \mathrm{~cm} \\
\mathrm{FR} & =0.5 \mathrm{~mL} \cdot \mathrm{h}^{-1}\end{aligned}$ & $\begin{array}{c}T=600-650-700-750^{\circ} \mathrm{C} \\
t=3 \mathrm{~h} \\
\mathrm{HR}=1^{\circ} \mathrm{C} \cdot \mathrm{min}^{-1}\end{array}$ & [26] \\
\hline $\mathrm{Fe}_{2} \mathrm{O}_{3}$ & $\mathrm{PVP} / \mathrm{Fe}\left(\mathrm{NO}_{3}\right)_{3} \cdot 9 \mathrm{H}_{2} \mathrm{O} /$ water/ethanol & $\begin{array}{l}V=30 \mathrm{kV} \\
D=15 \mathrm{~cm}\end{array}$ & $\begin{array}{c}T=500^{\circ} \mathrm{C} \\
t=4 \mathrm{~h} \\
\mathrm{HR}=1-7^{\circ} \mathrm{C} \cdot \mathrm{min}^{-1}\end{array}$ & [116] \\
\hline $\mathrm{MnO}_{2}$-doped $\mathrm{Fe}_{2} \mathrm{O}_{3}$ & Citric acid/ferric citrate/deionized water/manganese acetate & $\begin{aligned} V & =15 \mathrm{kV} \\
D & =10 \mathrm{~cm}\end{aligned}$ & $\begin{array}{c}T=400^{\circ} \mathrm{C} \\
\quad t=4 \mathrm{~h} \\
\mathrm{HR}=0.5^{\circ} \mathrm{C} \cdot \mathrm{min}^{-1}\end{array}$ & [117] \\
\hline $\mathrm{Fe}_{3} \mathrm{O}_{4} / \mathrm{Eu}(\mathrm{BA})_{3}$ phen/PVP & $\mathrm{Fe}_{3} \mathrm{O}_{4}$ nanoparticles/DMF/ $\mathrm{CHCl}_{3} / \mathrm{PVP} / \mathrm{Eu}(\mathrm{BA})_{3}$ phen powders & $\begin{aligned} V & =11 \mathrm{kV} \\
D & =12 \mathrm{~cm}\end{aligned}$ & & [45] \\
\hline $\mathrm{Tb}(\mathrm{BA})_{3}$ phen $/ \mathrm{PANI} / \mathrm{Fe}_{3} \mathrm{O}_{4} / \mathrm{PVP}$ & $\begin{array}{l}\text { Benzoic acid (BA)/phenan-throline (phen)/polyaniline (PANI) } / \mathrm{PVP} / \text { sulfonic } \\
\text { acid/ammonium persulfate/ethanol/CHCl } / \text { / DMF/nitric acid/deionized water } / \mathrm{Tb}_{4} \mathrm{O}_{7}\end{array}$ & $\begin{array}{c}V=13 \mathrm{kV} \\
D=14 \mathrm{~cm} \\
\mathrm{FR}=0.0167 \mathrm{~mL} \cdot \mathrm{min}^{-1}\end{array}$ & & [46] \\
\hline
\end{tabular}


Table 2. Cont

\begin{tabular}{|c|c|c|c|c|}
\hline Hollow Nanofiber & Precursors & $\begin{array}{l}\text { Electrospinning } \\
\text { Conditions }\end{array}$ & Annealing Conditions & Reference \\
\hline Carbon-coated $\mathrm{LiFePO}_{4}$ & $\begin{array}{l}\text { Lithium dihydrogen phosphate }\left(\mathrm{LiH}_{2} \mathrm{PO}_{4}\right) / \text { iron nitrate } \\
\text { 9-hydrate }\left(\left(\mathrm{Fe}\left(\mathrm{NO}_{3}\right)_{3} \cdot 9 \mathrm{H}_{2} \mathrm{O}\right) / \text { ferrous sulfate } 7 \text {-hydrate }\left(\mathrm{FeSO}_{4} \cdot 7 \mathrm{H}_{2} \mathrm{O}\right) / \text { DMF/PMMA }\right.\end{array}$ & $\begin{aligned} V & =16 \mathrm{kV} \\
D & =15 \mathrm{~cm} \\
\mathrm{FR} \text { (outer) } & =0.2 \mathrm{~mL} \cdot \mathrm{h}^{-1} \\
\mathrm{FR} \text { (inner) } & =0.4 \mathrm{~mL} \cdot \mathrm{h}^{-1}\end{aligned}$ & $\begin{array}{c}\mathrm{T}=7500^{\circ} \mathrm{C} \\
t=3 \mathrm{~h} \\
\mathrm{HR}=2{ }^{\circ} \mathrm{C} \cdot \mathrm{min}^{-1}\end{array}$ & [118] \\
\hline $\mathrm{CuO}$ & $\mathrm{PVP} /$ copper acetate $\left(\mathrm{Cu}\left(\mathrm{CH}_{3} \mathrm{COO}\right)_{2}\right) /$ ethanol & $\begin{aligned} V & =10 \mathrm{kV} \\
D & =13 \mathrm{~cm} \\
\mathrm{FR}= & 0.02 \mathrm{~mL} \cdot \mathrm{min}^{-1}\end{aligned}$ & $\begin{aligned} T & =500{ }^{\circ} \mathrm{C} \\
\quad t & =2 \mathrm{~h} \\
\mathrm{HR} & =6.7^{\circ} \mathrm{C} \cdot \mathrm{min}^{-1}\end{aligned}$ & [119] \\
\hline $\mathrm{CuO}$ & $\begin{array}{l}\text { Copper (II) sulfate pentahydrate } \\
\left(\mathrm{CuSO}_{4} \cdot 5 \mathrm{H}_{2} \mathrm{O}\right) / \mathrm{PVP} / \text { water }\end{array}$ & $\begin{array}{c}V=16.8 \mathrm{kV} \\
\mathrm{FR}=6 \mu \mathrm{L} \cdot \mathrm{min}^{-1}\end{array}$ & $\begin{array}{l}T=673 \text { and } 873 \mathrm{~K} \\
\quad t=5 \mathrm{~h}\end{array}$ & [120] \\
\hline $\mathrm{SnO}_{2}-\mathrm{ZnO}$ & $\mathrm{Zn}(\mathrm{AC})_{2} \cdot 2 \mathrm{H}_{2} \mathrm{O} / \mathrm{SnCl}_{2} \cdot 2 \mathrm{H}_{2} \mathrm{O} / \mathrm{PVP} / \mathrm{DMF} /$ ethanol/ethyl acetate & $\begin{aligned} V & =19 \mathrm{kV} \\
D & =20 \mathrm{~cm} \\
\mathrm{FR} & =0.7 \mathrm{~mL} \cdot \mathrm{h}^{-1}\end{aligned}$ & $\begin{array}{l}T=600{ }^{\circ} \mathrm{C} \\
t=3 \mathrm{~h}\end{array}$ & [121] \\
\hline $\mathrm{SnO}_{2}$ & Stannic chloride pentahydrate $\left(\mathrm{SnCl}_{4} \cdot 5 \mathrm{H}_{2} \mathrm{O}\right) /$ ethanol/DMF/PVP & $\begin{array}{l}\text { Electric field }=1.25 \mathrm{kV} / \mathrm{cm} \\
\quad D=18 \mathrm{~cm} \\
\mathrm{FR}=0.2 \mathrm{~mL} \cdot \mathrm{h}^{-1}\end{array}$ & $\begin{aligned} T= & 550-650^{\circ} \mathrm{C} \\
& t=4 \mathrm{~h}\end{aligned}$ & [122] \\
\hline Mn-Doped $\mathrm{SnO}_{2}$ & $\mathrm{SnCl}_{2} \cdot 2 \mathrm{H}_{2} \mathrm{O} / \mathrm{DMF} / \mathrm{ethanol} / \mathrm{PVP} / \mathrm{Mn}\left(\mathrm{CH}_{3} \mathrm{COO}\right)_{2} \cdot 4 \mathrm{H}_{2} \mathrm{O}$ & $\begin{aligned} V & =25 \mathrm{kV} \\
D & =18 \mathrm{~cm} \\
\mathrm{FR} & =1 \mathrm{~mL} \cdot \mathrm{h}^{-1}\end{aligned}$ & $\begin{array}{c}T=600{ }^{\circ} \mathrm{C} \\
t=3 \mathrm{~h}\end{array}$ & [123] \\
\hline Cerium-doped $\mathrm{SnO}_{2}$ & $\mathrm{SnCl}_{2} \cdot 2 \mathrm{H}_{2} \mathrm{O} / \mathrm{DMF} /$ ethanol/PVP/Ce $\left(\mathrm{NO}_{3}\right)_{3} \cdot 6 \mathrm{H}_{2} \mathrm{O}$ & $\begin{aligned} V & =25 \mathrm{kV} \\
D & =18 \mathrm{~cm}\end{aligned}$ & $\begin{aligned} T & =600^{\circ} \mathrm{C} \\
\quad t & =5 \mathrm{~h} \\
\mathrm{HR} & =5^{\circ} \mathrm{C} \cdot \mathrm{min}^{-1}\end{aligned}$ & [34] \\
\hline $\mathrm{Al}_{2} \mathrm{O}_{3}$ & Aluminum nitrate $\left(\mathrm{Al}\left(\mathrm{NO}_{3}\right)_{3}\right) / \mathrm{PAN} / \mathrm{DMF}$ & $\begin{aligned} V & =20 \mathrm{kV} \\
D & =20 \mathrm{~cm} \\
\mathrm{FR} & =1 \mathrm{~mL} \cdot \mathrm{h}^{-1}\end{aligned}$ & $\begin{array}{c}T=500-1000-1300^{\circ} \mathrm{C} \\
\mathrm{HR}=5^{\circ} \mathrm{C} \cdot \mathrm{min}^{-1}\end{array}$ & [54] \\
\hline$\gamma-\mathrm{Al}_{2} \mathrm{O}_{3}$ & Aluminum nitrate $\left(\mathrm{Al}\left(\mathrm{NO}_{3}\right)_{3}\right) / \mathrm{PAN} / \mathrm{DMF}$ & $\begin{aligned} V & =20 \mathrm{kV} \\
D & =20 \mathrm{~cm} \\
\mathrm{FR} & =1 \mathrm{~mL} \cdot \mathrm{h}^{-1}\end{aligned}$ & $\begin{aligned} T & =800^{\circ} \mathrm{C} \\
\quad t & =2 \mathrm{~h} \\
\mathrm{HR} & =5^{\circ} \mathrm{C} \cdot \mathrm{min}^{-1}\end{aligned}$ & [124] \\
\hline $\mathrm{Au} / \mathrm{V}_{2} \mathrm{O}_{5}$ & $\begin{array}{l}\text { Vanadyl acetylacetonate }\left(\mathrm{VO}(\mathrm{acac})_{2}\right) / \text { gold(III) chloride trihydrate } \\
\qquad\left(\mathrm{HAuCl}_{4} \cdot 3 \mathrm{H}_{2} \mathrm{O}\right) / \mathrm{PVP} / \text { polystyrene }(\mathrm{PS})\end{array}$ & $\begin{array}{c}V=20 \mathrm{kV} \\
D=20 \mathrm{~cm} \\
\mathrm{FR}=2 \mathrm{~mL} \cdot \mathrm{h}^{-1}\end{array}$ & $\begin{array}{c}T_{1}=330^{\circ} \mathrm{C} \\
t_{1}=2 \mathrm{~h} \\
\mathrm{HR}_{1}=5^{\circ} \mathrm{C} \cdot \mathrm{min}^{-1} \\
\mathrm{~T}_{2}=330-430^{\circ} \mathrm{C} \\
t_{2}=30 \mathrm{~min} \\
\mathrm{HR}_{2}=2^{\circ} \mathrm{C} \cdot \mathrm{min}^{-1}\end{array}$ & [50] \\
\hline
\end{tabular}


Table 2. Cont

\begin{tabular}{|c|c|c|c|c|}
\hline Hollow Nanofiber & Precursors & $\begin{array}{l}\text { Electrospinning } \\
\text { Conditions }\end{array}$ & Annealing Conditions & Reference \\
\hline Vanadium nitride (VN) & $\begin{array}{l}\text { Oxalic acid dihydrate }\left(\mathrm{C}_{2} \mathrm{H}_{2} \mathrm{O}_{4} \cdot 2 \mathrm{H}_{2} \mathrm{O}\right) / \text { ethanol/PVP/ammonium } \\
\text { metavanadate }\left(\mathrm{NH}_{4} \mathrm{VO}_{3}\right)\end{array}$ & $\begin{array}{l}V=15 \mathrm{kV} \\
D=15 \mathrm{~cm}\end{array}$ & $\begin{array}{c}T=400-600-800{ }^{\circ} \mathrm{C} \\
\quad t=1 \mathrm{~h} \\
\mathrm{HR}=2^{\circ} \mathrm{C} \cdot \min ^{-1}\end{array}$ & [125] \\
\hline $\mathrm{CNTs} / \mathrm{InVO}_{4}$ & Multi-walled carbon nanotubes/ $\mathrm{In}\left(\mathrm{NO}_{3}\right)_{3} \cdot 4.5 \mathrm{H}_{2} \mathrm{O} / \mathrm{C}_{10} \mathrm{H}_{14} \mathrm{O}_{5} \mathrm{~V} / \mathrm{PVP} /$ ethanol & $\begin{aligned} V & =21 \mathrm{kV} \\
D & =15 \mathrm{~cm}\end{aligned}$ & $\begin{aligned} T & =550{ }^{\circ} \mathrm{C} \\
t & =2 \mathrm{~h}\end{aligned}$ & [126] \\
\hline $\mathrm{Te}$ & $\mathrm{Ni}$ acetate/PVP/HTeO ${ }^{2+}$ & & & [127] \\
\hline $\mathrm{LiFePO}_{4} / \mathrm{C} / \mathrm{Ag}$ & $\mathrm{Fe}\left(\mathrm{NO}_{3}\right)_{3} \cdot 9 \mathrm{H}_{2} \mathrm{O} / \mathrm{AgNO}_{3} / \mathrm{H}_{3} \mathrm{PO}_{4} / \mathrm{LiOH} \cdot \mathrm{H}_{2} \mathrm{O} / \mathrm{DMF} / \mathrm{PVP}$ & $\begin{array}{l}V=13 \mathrm{kV} \\
D=16 \mathrm{~cm}\end{array}$ & $\begin{array}{c}T=700^{\circ} \mathrm{C} \\
\quad t=10 \mathrm{~h} \\
\mathrm{HR}=1{ }^{\circ} \mathrm{C} \cdot \min ^{-1}\end{array}$ & [128] \\
\hline Chromium-doped spinel & $\begin{array}{c}\mathrm{Zn}\left(\mathrm{NO}_{3}\right)_{2} \cdot 6 \mathrm{H}_{2} \mathrm{O} / \mathrm{Mg}\left(\mathrm{NO}_{3}\right)_{2} \cdot 6 \mathrm{H}_{2} \mathrm{O} / \mathrm{Al}\left(\mathrm{NO}_{3}\right)_{3} \cdot 9 \mathrm{H}_{2} \mathrm{O} / \mathrm{Cr}\left(\mathrm{NO}_{3}\right)_{3} \cdot 9 \mathrm{H}_{2} \mathrm{O} / \text { ethanol/ } \\
\text { deionized water/PVP }\end{array}$ & $\begin{aligned} V & =20 \mathrm{kV} \\
D & =12 \mathrm{~cm} \\
\mathrm{FR} & =1.5 \mathrm{~mL} \cdot \mathrm{h}^{-1}\end{aligned}$ & $\begin{array}{c}T=1000-1100-1200^{\circ} \mathrm{C} \\
\quad t=5 \mathrm{~h} \\
\operatorname{HR}=200^{\circ} \mathrm{C} \cdot \mathrm{h}^{-1}\end{array}$ & [129] \\
\hline $\mathrm{YF}_{3}: \mathrm{Eu}^{3+}$ & $\begin{array}{l}\text { Yttrium oxide }\left(\mathrm{Y}_{2} \mathrm{O}_{3}\right) / \text { europium oxide }\left(\mathrm{Eu}_{2} \mathrm{O}_{3}\right) / \mathrm{DMF} / \text { ammonium hydrogen } \\
\text { fluoride }\left(\mathrm{NH}_{4} \mathrm{HF}_{2}\right) / \text { Nitric acid }\left(\mathrm{HNO}_{3}\right) / \text { ethyl alcohol }\end{array}$ & $\begin{aligned} V & =13 \mathrm{kV} \\
D & =16 \mathrm{~cm}\end{aligned}$ & $\begin{array}{c}T_{1}=700^{\circ} \mathrm{C} \\
t_{1}=8 \mathrm{~h} \\
\mathrm{HR}_{1}=1^{\circ} \mathrm{C} \cdot \mathrm{min}^{-1} \\
T_{2}=200^{\circ} \mathrm{C} \\
\mathrm{HR}_{2}=1^{\circ} \mathrm{C} \cdot \mathrm{min}^{-1}\end{array}$ & [130] \\
\hline $\mathrm{YF}_{3}: \mathrm{Yb}^{3+} / \mathrm{Er}^{3+}$ & $\begin{array}{l}\text { Yttrium oxide }\left(\mathrm{Y}_{2} \mathrm{O}_{3}\right) / \text { erbium oxide }\left(\mathrm{Er}_{2} \mathrm{O}_{3}\right) / \mathrm{PVP} / \mathrm{DMF} / \text { ammonium hydrogen } \\
\text { fluoride }\left(\mathrm{NH}_{4} \mathrm{HF}_{2}\right) / \text { Nitric acid }\left(\mathrm{HNO}_{3}\right) /\end{array}$ & $\begin{array}{l}V=16 \mathrm{kV} \\
D=18 \mathrm{~cm}\end{array}$ & $\begin{array}{c}T_{1}=700^{\circ} \mathrm{C} \\
t_{1}=8 \mathrm{~h} \\
\mathrm{HR}_{1}=1{ }^{\circ} \mathrm{C} \cdot \mathrm{min}^{-1} \\
T_{2}=200{ }^{\circ} \mathrm{C} \\
\mathrm{HR}_{2}=1^{\circ} \mathrm{C} \cdot \mathrm{min}^{-1}\end{array}$ & [35] \\
\hline
\end{tabular}

$V=$ voltage, $D=$ distance between nozzle to collector, $\mathrm{FR}=$ flow rate, $T=$ temperature, $t=$ time, $\mathrm{HR}=$ heating rate 


\subsection{Titanium Dioxide $\left(\mathrm{TiO}_{2}\right)$ Hollow Nanofibers for Photodecomposition of Organic Pollutants}

$\mathrm{TiO}_{2}$ is a semiconducting material with promising characteristics, such as a long-standing stability against chemical and photo corrosion. It is environmentally friendly and shows great photo-reactivity, robust oxidizing activity, and good optical transparency. Moreover, optimum dielectric properties and electrical conductivity, a large refractive index of about 2.52 for anatase and 2.49 for rutile, as well as a large band gap of 3-3.5 eV are other interesting features of $\mathrm{TiO}_{2}$ [131]. Thanks to such unique properties, it has been considered for a wide range of applications including solar cells, environmental protection and cleaning, sensors, photocatalysis, photoelectronics, etc. [9,131-134].

As a result of the wide band gap, $\mathrm{TiO}_{2}$ absorbs Ultraviolet (UV) light and shows optimum photocatalytic activity. This ability is enhanced on large surface areas, e.g., hollow nanostructures, and brings about an efficient degradation of inorganic and organic molecules [135]. In fact, a large surface size leads to a rapid charging-discharging rate because of the small diffusion length and high surface area [136]. In this regard, Zhao et al. [48] introduced nanosized interior hollow channels into $\mathrm{TiO}_{2}$ microfibers. The multichannel structured fibers were synthesized by a multifluidic compound-jet electrospinning method (Figure 6a). The optimized photocatalytic activity of the fibers was employed for the degradation of acetaldehyde gas. The authors concluded that the multichannel structure of hollow $\mathrm{TiO}_{2}$ fibers (Figure $6 \mathrm{~b}$ ) causes two effects: a multiple reflection of the incident light, and an inner entrapment of the gaseous molecules. As shown in Figure $6 c$, the photocatalytic degradation of acetaldehyde by $\mathrm{TiO}_{2}$ is done via a reaction with first-order kinetics. This fact is confirmed by the linear plot of $\ln \left(C_{0} / C_{t}\right)$ versus the photocatalytic reaction time $t$. While $C_{0}$ is the initial concentration of acetaldehyde, $C_{t}$ is the concentration of acetaldehyde after the photocatalytic reaction for $t$ in hours. This Figure implies that the initial rate constant $(k)$ for acetaldehyde degradation increases proportionally to the channel number from solid fibers $\left(k_{0 \mathrm{CF}} \approx 0.37 \mathrm{~h}^{-1}\right)$, one-channel fibers $\left(k_{1 \mathrm{CF}} \approx 0.40 \mathrm{~h}^{-1}\right)$, two-channel fibers $\left(k_{2 \mathrm{CF}} \approx 0.47 \mathrm{~h}^{-1}\right)$, to three-channel fibers $\left(k_{3 \mathrm{CF}} \approx 0.83 \mathrm{~h}^{-1}\right)$. Hollow $\mathrm{TiO}_{2}$ nanofibers with a promising photocatalytic effect for the decomposition of the methylene blue (MB) dye have also been synthesized by Chang et al. [47] via coaxial electrospinning. For this, they used titanium sol (a mixture of ethanol, acetic acid, PVP, and titanium butoxide (TBT)) and a titanium precursor (a mixture of TBT and ethylene glycol) for the shell, to produce two types of hollow crystalline $\mathrm{TiO}_{2}$ nanofibers. The core fluid was solely a PVP/ethanol/deionized water solution. Both types of nanofibers showed a similar tubular structure. Yet, they were different in terms of surface morphology and shell thickness. When titanium sol was used, a small amount of water in the core hampered the diffusion of the core and shell solutions. Since the titanium precursor is not spinnable, PVP nanofibers were used as core and shell templates during the coaxial electrospinning process. Both hollow $\mathrm{TiO}_{2}$ nanofibers were superior to their solid counterparts in terms of $\mathrm{MB}$ dye degradation efficiency. In this context, Jung et al. [110] also produced multiwalled carbon nanotube (MWCNT)-embedded $\mathrm{TiO}_{2}$ hollow nanofibers that could efficiently photodegrade MB. They stated that the improved degradation efficiency is due to electrons transfer between $\mathrm{TiO}_{2}$ and $\mathrm{MWCNT}$ and to the MWCNT adsorption ability. The maximum MB decomposition rate of $62 \%$ was obtained after $70 \mathrm{~min}$. Regarding another composite hollow nanofiber system, Peng et al. [106] reported that the photocatalytic activity of hollow $\mathrm{SnO}_{2} / \mathrm{TiO}_{2}$ nanofibers prepared by coaxial electrospinning is greater than that of the commercial $\mathrm{TiO}_{2}$ photocatalysts. This could be due to their one-dimensional hollow structure and to a continuous hetero-junction between $\mathrm{TiO}_{2}$ and $\mathrm{SnO}_{2}$. The $\mathrm{SnO}_{2} / \mathrm{TiO}_{2}$ hollow nanofibers could decompose Rhodamine- $\mathrm{B}(\mathrm{RhB})$ dye faster than solid $\mathrm{TiO}_{2}$ nanofibers and $\mathrm{TiO}_{2}$ nanoparticles did. 

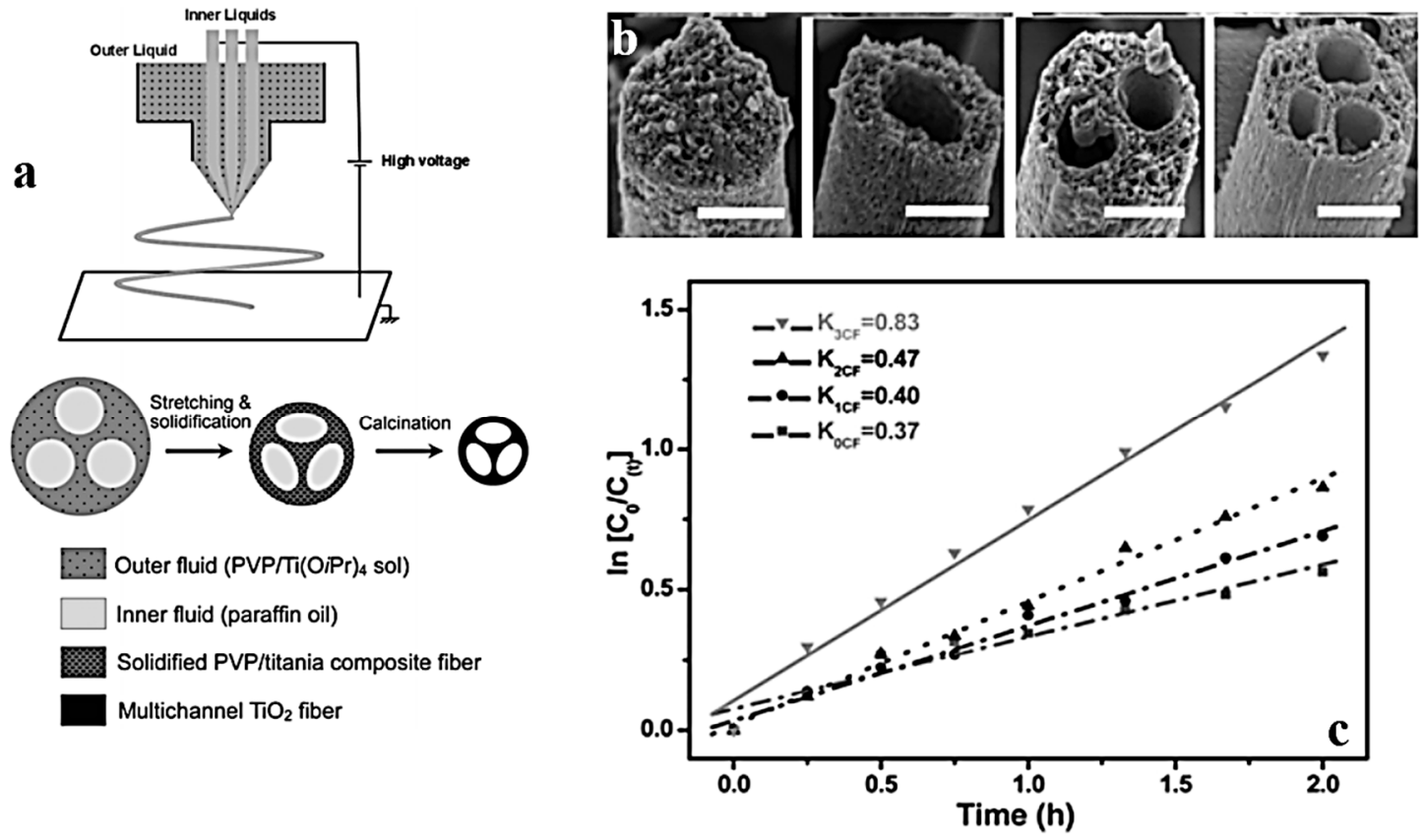

Figure 6. (a) The schematic illustration of a multifluidic compound-jet electrospinning method wherein the set-up consists of an outer nozzle and three inner capillaries; (b) SEM images of $\mathrm{TiO}_{2}$ fibers with $0,1,2$, and 3 channels (from left to right). The scale bar is $1 \mu \mathrm{m}$; (c) The multichannel structure of the hollow $\mathrm{TiO}_{2}$ fibers enhances the kinetics of the degradation process of acetaldehyde gas (Reproduced with permission from [48]. Royal Society of Chemistry, 2017).

In another research, Hou et al. [55] produced mesoporous walled $\mathrm{TiO}_{2}$ hollow nanofibers whose composition consisted of rutile (5.4\%) and anatase (94.6\%). To confer mesoporosity to the nanofibers, a foaming agent was added to the mixed-phase composition. Accordingly, the hollow structure and the mesoporous walls of the nanofibers could cooperatively bring about an excellent photocatalytic efficiency and stability. The as-synthesized nanofibers, whose inner diameter and wall thickness were 215 and $100 \mathrm{~nm}$, respectively, were able to decompose RhB efficiently (99.5\%) in $60 \mathrm{~min}$.

To shorten the band gap of $\mathrm{TiO}_{2}$ in order to extend its photocatalytic applicability to visible light, Yang et al. [112] loaded hollow $\mathrm{TiO}_{2}$ nanofibers with Pt nanoparticles. The schematic of the entire process, including electrospinning, calcination, and Pt loading of the nanofibers, is demonstrated in Figure 7a. Moreover, the morphology of the as-synthesized $\mathrm{Pt} / \mathrm{TiO}_{2}$ hollow nanofiber is depicted in Figure $7 \mathrm{~b}$. The nanofibers were made of an anatase-rutile (70:30) mixed phase. By inclusion of $\mathrm{Pt}(2 \mathrm{wt}$. \%), the band gap of the hollow nanofibers declined from 3.09 to $2.77 \mathrm{eV}$. This modification resulted in the possibility of a photocatalytic process under visible light. Such a system was studied in terms of degradation of the azo dye orange II. The results represented in Figure 7c imply that such a doped hollow nanofiber is able to degrade the dye molecules with a pseudo-first-rate constant of $0.0069 \mathrm{~min}^{-1}$, which was 11.5 and 3.63 times larger than that for the unloaded hollow nanofibers and $\mathrm{Pt} / \mathrm{P} 25$ ( $\mathrm{TiO}_{2}$ nanoparticles), respectively. The factors affecting this performance included the $\mathrm{Pt}$ loading amount, the calcination temperature of the $\mathrm{TiO}_{2}$ hollow nanofibers, the $\mathrm{pH}$ of the primary solution, and the light source. The results demonstrated that by addition of $2 \mathrm{wt}$. \% Pt, calcination of the nanofibers at $350^{\circ} \mathrm{C}$, and application of the nanofibers in acidic condition and under solar light, the best photocatalytic activity can be achieved. The main decomposition mechanism of Orange II was attributed to the oxidation by $\mathrm{H}^{+}$and $\mathrm{O}_{2}{ }^{-}$radicals, as shown in Figure $7 \mathrm{~d}$. 


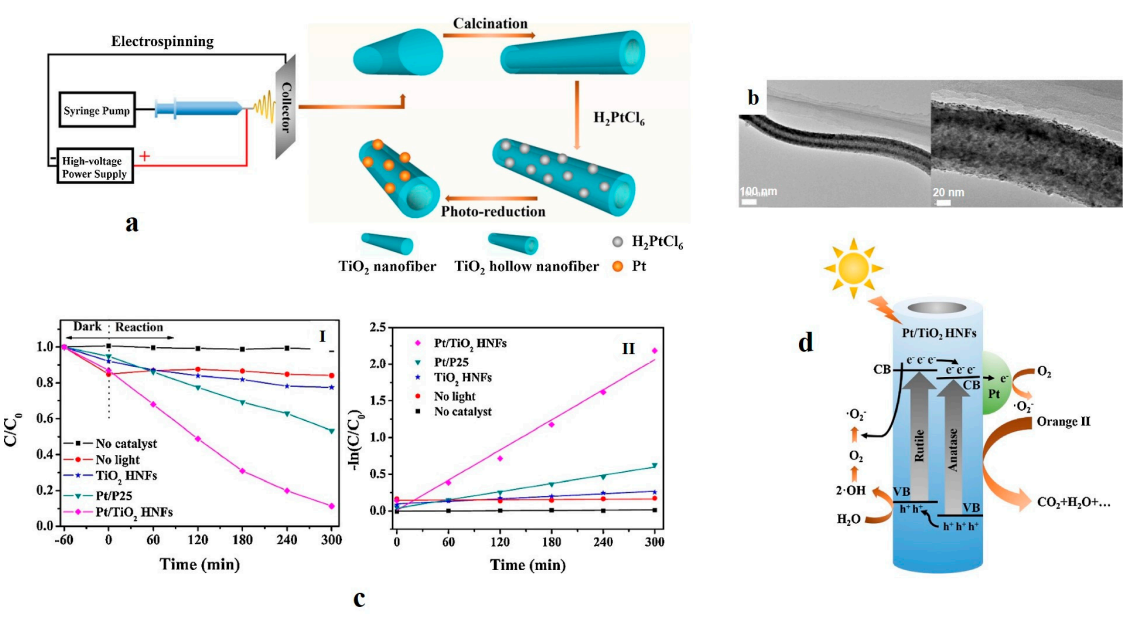

Figure 7. (a) The schematic illustration of the entire process of fabrication of $\mathrm{Pt} / \mathrm{TiO}_{2}$ hollow nanofibers (HNFs); (b) TEM image of the $2 \mathrm{wt} \% \mathrm{Pt} / \mathrm{TiO}_{2}$ hollow nanofiber calcined at $350{ }^{\circ} \mathrm{C}$ at two magnifications (the scale bars are 100 and $20 \mathrm{~nm}$ ); (c) Photodegradation of Orange II under visible light by various photocatalysts (I) and kinetic graphs relevant to the photodegradation of Orange II (II); (d) Schematic illustration of the photodegradation process of Orange II (Reproduced with permission from [112]. Elsevier, 2017).

\subsection{Ferrite Hollow Nanofibers for Electromagnetic and Photocatalytic Devices}

Ferrites are natural, abundant, inexpensive, and sustainably permanent magnets. They are known for their spinel shape and the common formula of $\mathrm{MFe}_{2} \mathrm{O}_{4}$, in which $\mathrm{M}$ stands for $\mathrm{Fe}, \mathrm{Co}, \mathrm{Ni}$, or $\mathrm{Mn}$. They show interesting magneto-optical, magnetic, electrical, and magnetoresistive characteristics [137,138]. Such features suggest them as promising candidates for electronic devices (e.g., antenna rod, computer components, and memory devices) [139], batteries, sensors, magnetic recording media, magnetic drug delivery, and catalysis [140].

Among various ferrites, the one based on cobalt $(\mathrm{Co})$ has received considerable attention especially for magnetic recording applications because of its anisotropy, optimum chemical stability, mechanical hardness, saturation magnetization, and high coercivity [141,142]. The magnetic properties of this kind of ferrites are influenced by purity, size, and shape of the material, and hence by the manufacturing method [143]. Therefore, it is assumed that a hollow nanostructured ferrite could bring about promising magnetic property. In this regard, Cheng et al. [113] produced $\mathrm{CoFe}_{2} \mathrm{O}_{4}$ hollow nanofibers via electrospinning of a PVP/nitrate salts solution and subsequent calcination. The as-synthesized nanofibers were characterized in terms of crystalline structure, morphology, magnetic properties, etc. The results of X-ray diffraction analysis (XRD), SEM and TEM confirmed the cubic spinel structure, one-dimensional texture, and the existence of many nanoparticles in the wall of the hollow fibers, respectively.

In solution, the carbonyl and tertiary amine groups of PVP can coordinate with $\mathrm{Fe}^{3+}$ and $\mathrm{Co}^{2+}$, thereby forming a metal-organic framework structure. During the electrospinning process, by evaporation of the solvent (here ethanol/water) the composite fibers are created and can then act as a self-sacrificial template for the $\mathrm{CoFe}_{2} \mathrm{O}_{4}$ hollow nanofibers. Subsequently, the composite fibers are converted to the hollow nanofibers. The mechanism of formation of the hollow nanofibers could be related to the diffusion of the gas product of the PVP decomposition, driving the nanoparticles constituting the fibers from the inside to the outside of the composite fibers.

As shown in this study, the $\mathrm{CoFe}_{2} \mathrm{O}_{4}$ hollow fibers show a typical ferromagnetic (FM) behavior. As a definition, saturation magnetization $\left(M_{\mathrm{S}}\right)$ is the highest induced magnetic moment that a material can get in a magnetic field. As the authors report, the $M_{\mathrm{s}}$ value of the $\mathrm{CoFe}_{2} \mathrm{O}_{4}$ hollow fibers increases with increasing annealing temperatures. The hollow fibers synthesized in this study are made of many nanoparticles, whose interactions and properties determine the magnetic behavior 
of the $\mathrm{CoFe}_{2} \mathrm{O}_{4}$ hollow fibers. For small $\mathrm{CoFe}_{2} \mathrm{O}_{4}$ nanoparticles, the inner sides of the nanoparticles are in the usual spin arrangement, whereas the arrangement of their surface atomic moments is disordered. This discrepancy could break their surface exchange bond and change their surface cations coordination, thus decreasing the $\mathrm{M}_{\mathrm{s}}$ of the $\mathrm{CoFe}_{2} \mathrm{O}_{4}$ nanoparticles. As a fact, the higher the calcination temperature is, the larger the particle size will be. Thus, the surface shell contribution to the $M_{\mathrm{s}}$ would decline when the nanoparticles' size increases. This means that the $M_{\mathrm{S}}$ would increase at higher annealing temperatures. As shown in this study, at the higher calcination temperature of $700{ }^{\circ} \mathrm{C}$, $M_{\mathrm{S}}$ for the $\mathrm{CoFe}_{2} \mathrm{O}_{4}$ hollow fibers is $34.71 \mathrm{emu} \cdot \mathrm{g}^{-1}$ at $300 \mathrm{~K}$, and $36.82 \mathrm{emu} \cdot \mathrm{g}^{-1}$ at $2 \mathrm{~K}$, respectively. This difference can be attributed to the surface spin-canting effect and to the existence of a magnetic dead layer on the surface. When the temperature is low, the surface spins will be frozen along, and thus the local surface anisotropy will be higher. However, at high applied fields, the surface anisotropy once again decreases, and the surface spins are arranged along the field direction, leading to a higher $M_{\mathrm{s}}$ magnitude. In general, the magnetic measurements verified that the $\mathrm{CoFe}_{2} \mathrm{O}_{4}$ hollow nanofibers are able to offer novel magnetic properties that are promising for electromagnetic and spintronic devices.

Other than their electromagnetic properties, $\mathrm{CoFe}_{2} \mathrm{O}_{4}$ hollow nanofibers could also be employed in connection with photocatalytic applications. In this regard, Kim et al. [115] produced hollow core-double-sheath nanofibers with a $\mathrm{CoFe}_{2} \mathrm{O}_{4}$ internal sheath and a PANI external sheath through electrospinning, annealing, and in situ chemical oxidative polymerization. The mechanism of formation of such nanofibers is demonstrated in Figure 8a. The hollow nanofibers were mesoporous, with improved electrical conductivity and optical properties. The hetero-junction made between $\mathrm{CoFe}_{2} \mathrm{O}_{4}$ and PANI enhanced visible light photocatalysis. The authors concluded that the hollow $\mathrm{CoFe}_{2} \mathrm{O}_{4}$-PANI nanofibers harvest visible light, make the quantum confinement impact, assist the electron and mass transfer, and support the charge separation. Thanks to the unique core-shell mesoporous structure of the $\mathrm{CoFe}_{2} \mathrm{O}_{4}$-PANI nanofibers, the pseudo-first-order kinetic constant of the photocatalytic degradation of the methyl orange (MO) dye under visible light was 80 times larger than that for the $\mathrm{CoFe}_{2} \mathrm{O}_{4}$ nanofibers. This finding, along with the photodegradation efficiency of the mentioned nanofibers, is presented in Figure 8b. As shown in Figure 8c, the notably improved photocatalytic activity of the $\mathrm{CoFe}_{2} \mathrm{O}_{4}$-PANI nanofibers is because of the interaction and synergistic effects of $\mathrm{CoFe}_{2} \mathrm{O}_{4}$ and PANI, optimally inducing the separation of electron-hole in the $\mathrm{CoFe}_{2} \mathrm{O}_{4}$ and PANI coupling system.
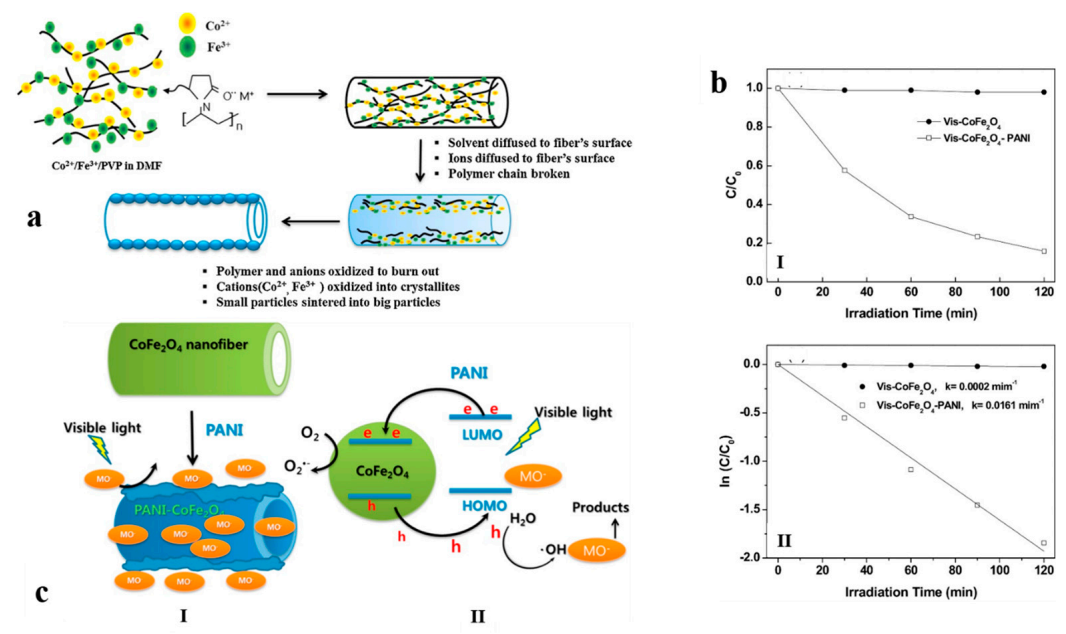

Figure 8. (a) Schematic illustration of the formation process of $\mathrm{CoFe}_{2} \mathrm{O}_{4}$ hollow nanofibers; (b) (I) visible light photodegradation and (II) kinetic linear simulation curves of the methyl orange (MO) dye for $\mathrm{CoFe}_{2} \mathrm{O}_{4}$ and $\mathrm{CoFe}_{2} \mathrm{O}_{4}-\mathrm{PANI}$ hollow nanofibers; (c) (I) schematic diagram and (II) mechanism of the photodegradation process by $\mathrm{CoFe}_{2} \mathrm{O}_{4}$-PANI hollow nanofiber when subjected to visible light (Reproduced with permission from [115]. Elsevier, 2017). 


\subsection{Iron Compound-Based Hollow Nanofibers for Ferromagnetic Devices}

Among the eight known types of iron oxides and different transition metal oxides, magnetite $\left(\mathrm{Fe}_{3} \mathrm{O}_{4}\right)$, hematite $\left(\alpha-\mathrm{Fe}_{2} \mathrm{O}_{3}\right)$, and maghemite $\left(\gamma-\mathrm{Fe}_{2} \mathrm{O}_{3}\right)$ are widely applied because of their catalytic, biomedical, and magnetic properties. Moreover, their abundance, low-cost processing, lack of toxicity, great theoretical capacity $\left(\approx 1000 \mathrm{~mA} \cdot \mathrm{h} \cdot \mathrm{g}^{-1}\right)$, and corrosion resistance are promising for a diverse range of applications [144-146].

In comparison with other iron oxide polymorphs, $\alpha-\mathrm{Fe}_{2} \mathrm{O}_{3}$ has been widely utilized in batteries, sensors, electrodes, magnetic resonance imaging, transistors, and supercapacitors [147]. To induce a high ferromagnetic property, Cheng et al. [116] synthesized $\mathrm{Fe}_{2} \mathrm{O}_{3}$ hollow nanofibers by calcination of electrospun PVP $/ \mathrm{Fe}\left(\mathrm{NO}_{3}\right)_{3}$ nanofibers at $500{ }^{\circ} \mathrm{C}$ for $4 \mathrm{~h}$, with the heating rate of $1-7^{\circ} \mathrm{C} \cdot \mathrm{min}^{-1}$. By changing the production conditions, the morphology of $\mathrm{Fe}_{2} \mathrm{O}_{3}$ changes from solid belt to hollow belts and hollow fibers. They found that the key parameter for the hollow nanostructure formation is the PVP decomposition rate, which can be controlled by tuning the heating rate and the rigidity of the surface gel layer. The latter can be changed by the solvent composition as well as by the PVP and $\mathrm{Fe}\left(\mathrm{NO}_{3}\right)_{3} \cdot 9 \mathrm{H}_{2} \mathrm{O}$ content. The authors also investigated the magnetic properties of hollow fibers at room temperature, with the field sweeping from -15 to $+15 \mathrm{kOe}$. The hollow fibers remnant magnetization $\left(M_{r}\right)$ and coercivity $\left(H_{c}\right)$ are $0.13 \mathrm{emu} \cdot \mathrm{g}^{-1}$ and $177.4 \mathrm{Oe}$, respectively. These values imply that the hollow fibers show ferromagnetic behaviors at room temperature. Compared to the urchin-like, rod-like, and rhombohedral $\mathrm{Fe}_{2} \mathrm{O}_{3}$ counterparts, the reported values of $M_{\mathrm{r}}$ and $H_{\mathrm{c}}$ of the hollow fibers are higher $\left(4.678 \times 10^{-3}, 2.754 \times 10^{-3}\right.$, and $1.043 \times 10^{-3} \mathrm{emu} \cdot \mathrm{g}^{-1}$ for $M_{\mathrm{r}}, 92.235,46.94$, and 77.75 Oe for $H_{c}$, respectively). The reason should be sought in the sensitivity of the magnetization of the ferromagnetic materials to the morphology and structure of the samples made thereof. Here, the assembly of the nanoparticles into the hollow fibers can convert a single domain to a multidomain, thus increasing the $M_{\mathrm{r}}$ and $H_{\mathrm{c}}$ values.

$\mathrm{Fe}_{3} \mathrm{O}_{4}$ shows the strongest magnetism among the transition metal oxides. Also, it offers optimum biocompatibility and low level of cytotoxicity in living cells [144]. The preparation of multifunctional hollow nanofibers including a $\mathrm{Fe}_{3} \mathrm{O}_{4}$ component via one-pot coaxial electrospinning without a core solution (using air only), as shown in Figure 9a, has been frequently studied $[45,46,148]$. For instance, $\mathrm{Yu}$ et al. [45] fabricated bifunctional, magnetic luminescent $\mathrm{Fe}_{3} \mathrm{O}_{4} / \mathrm{Eu}(\mathrm{BA})_{3}$ phen/PVP hollow nanofibers. In another work, Liu et al. [148] constructed trifunctional luminescent-electrical-magnetic $\mathrm{Eu}(\mathrm{BA})_{3}$ phen/PANI/ $\mathrm{Fe}_{3} \mathrm{O}_{4} / \mathrm{PVP}$ hollow nanofibers (Figure 9b,c). The electrical conductivity of $10^{-3} \mathrm{~S} \cdot \mathrm{cm}^{-1}$ was reported for these hollow nanofibers. The fluorescence emission peaks of $\mathrm{Eu}^{3+}$ were detected in the hollow nanofibers and attributed to the ${ }^{5} \mathrm{D}_{0} \rightarrow{ }^{7} \mathrm{~F}_{0}(580 \mathrm{~nm}),{ }^{5} \mathrm{D}_{0} \rightarrow{ }^{7} \mathrm{~F}_{1}$ (592 nm), and ${ }^{5} \mathrm{D}_{0} \rightarrow{ }^{7} \mathrm{~F}_{2}(616 \mathrm{~nm})$ energy level transitions of $\mathrm{Eu}^{3+}$ ions. The latter hypersensitive transition induces the strongest emission peak. Interestingly, the luminescent intensity, electrical conductivity, and magnetic properties of the hollow nanofibers were proportional to the amount of $\mathrm{Eu}(\mathrm{BA})_{3}$ phen, PANI and $\mathrm{Fe}_{3} \mathrm{O}_{4}$ nanoparticles, respectively, and could be adjusted. The photoluminescent-electrical-magnetic trifunctional flexible hollow nanofibers can be proposed for diverse applications, such as electromagnetic interference shielding, microwave absorption, molecular electronics, and biomedicine. 


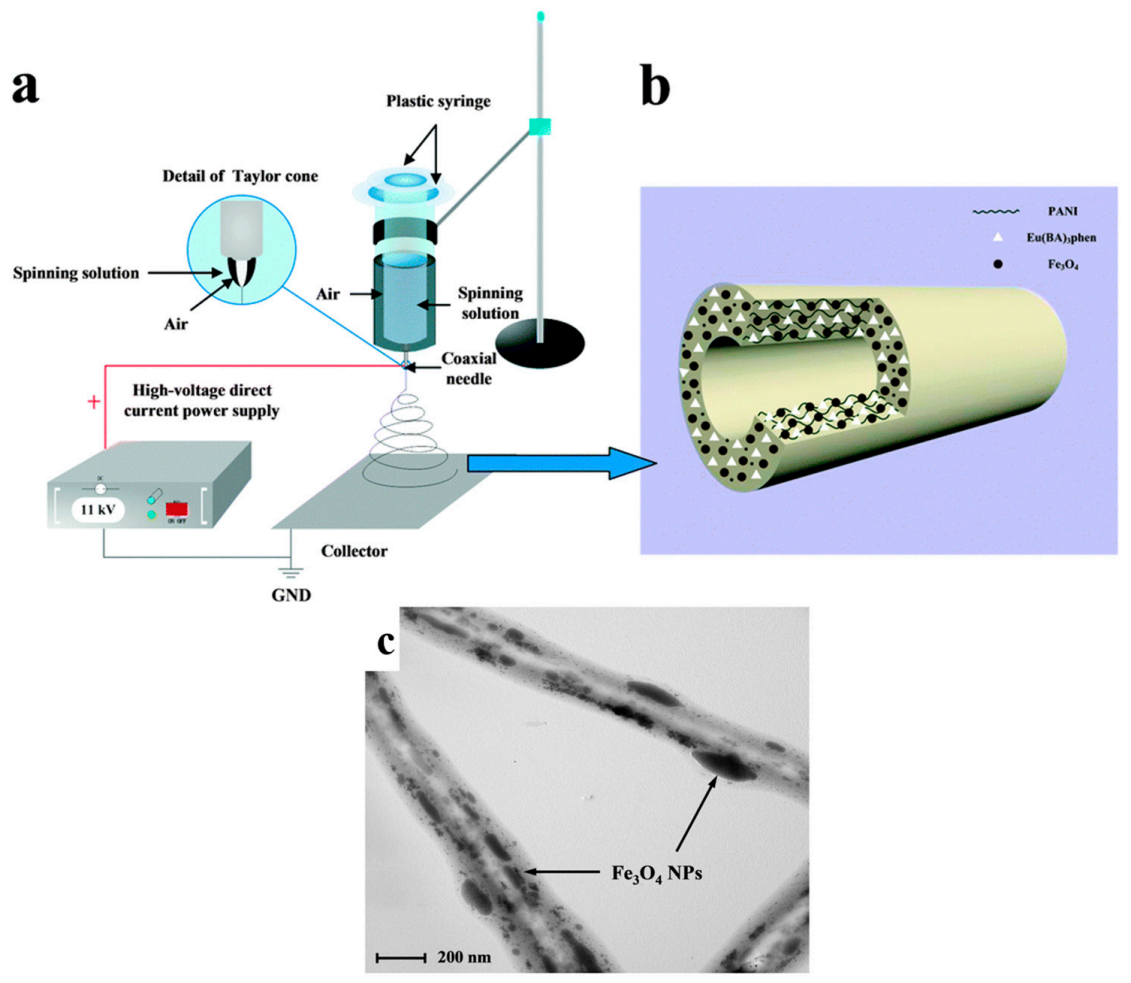

Figure 9. (a) The schematic shows details of the one-pot coaxial electrospinning process and set-up; (b) the schematic of the as-synthesized hollow nanofibers containing the europium complex, PANI, and $\mathrm{Fe}_{3} \mathrm{O}_{4}$ nanoparticles; (c) TEM image of the $\mathrm{Eu}(\mathrm{BA})_{3}$ phen/PANI/ $\mathrm{Fe}_{3} \mathrm{O}_{4} / \mathrm{PVP}$ hollow nanofibers (Reproduced with permission from [148]. Royal Society of Chemistry, 2017).

\subsection{Zinc Oxide Hollow Nanofibers for Gas Sensing}

Zinc oxide $(\mathrm{ZnO})$ is a semiconductor with the band gap energy of $3.37 \mathrm{eV}$, a large exciton binding energy of $60 \mathrm{meV}$, excellent chemical and thermal stabilities, and high transparency. Such features enable its use for a wide variety of applications, such as solar cells, sensors, photodetectors, transistors, etc. [149-151]. Additionally, $\mathrm{ZnO}$ provides a high adsorption capacity for the removal of heavy metals [152], as well as a long life span and great ultraviolet absorption [153]. Electrospun ZnO nanofibers exhibit notable optoelectronic, catalytic, humidity sensing, and piezoelectric properties, as well as a great sensitivity to different gases (e.g., $\mathrm{NO}_{2}, \mathrm{H}_{2}, \mathrm{CO}, \mathrm{C}_{2} \mathrm{H}_{5} \mathrm{OH}, \mathrm{H}_{2} \mathrm{~S}$, and $\mathrm{NH}_{3}$ ) [154]. A much larger surface area induced by a hollow structure can notably enhance such promising features. For example, Zhang et al. [20] developed $\mathrm{ZnO}$ hollow nanofibers via single-spinneret electrospinning of a precursor solution composed of PAN, PVP, and zinc acetate. The composite nanofibers subsequently underwent thermal decomposition to eliminate the polymers and to fabricate $\mathrm{ZnO}$ hollow nanofibers. This process is schematically shown in Figure 10a. During the electrospinning process, a phase separation occurs so that the precursor nanofibers of PAN, PVP, and zinc acetate composite show a core-shell structure consisting of a PAN core and a PVP/zinc acetate composite shell. The as-synthesized $\mathrm{ZnO}$ hollow nanofibers can offer remarkable sensing properties against ethanol because of their special one-dimensional hollow nanostructure with an extensive surface area. The sensitivity (S) of the sensor follows the equation $S=R_{\mathrm{a}} / R_{\mathrm{g}}$, where $R_{\mathrm{a}}$ and $R_{\mathrm{g}}$ are the sensor resistance in atmospheric air and in ethanol-air mixed gas, respectively. The response and recovery time are the times spent by the sensor to achieve $90 \%$ of the total resistance change in the case of adsorption and desorption, respectively. 

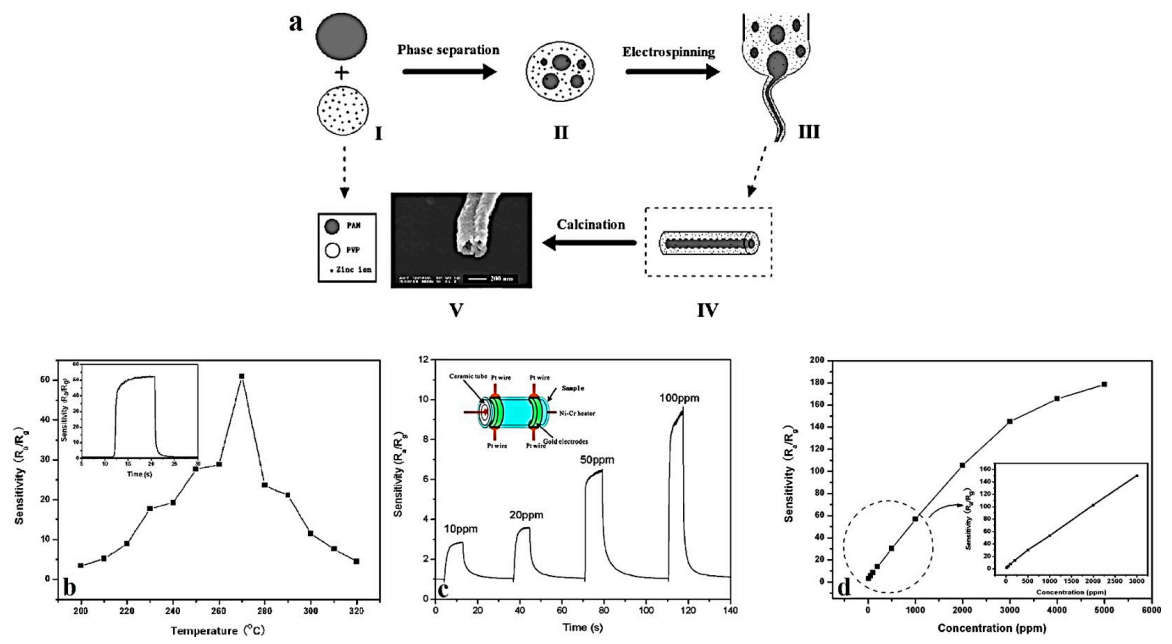

Figure 10. (a) The schematic demonstration of various steps of the formation process of $\mathrm{ZnO}$ hollow nanofibers; (b) ethanol sensitivity of the $\mathrm{ZnO}$ hollow nanofibers at different operating temperatures (the inset shows the response-recovery curve of the nanofibers exposed to $1000 \mathrm{ppm}$ of ethanol); (c) dynamic response of the sensor to ethanol, whose concentration varies from 10 to $100 \mathrm{ppm}$ (the inset image shows, schematically, the $\mathrm{ZnO}$ hollow nanofibers sensor connected to the electrodes); (d) the sensor sensitivity versus ethanol concentration (the inset graph implies a linear relationship between sensitivity and ethanol concentration) (Reproduced with permission from [20]. American Chemical Society, 2017).

In the ethanol sensing process by the $\mathrm{ZnO}$ hollow nanofibers, oxygen sorption adversely contributes to the electrical transport properties of the nanofibers. When oxygen is adsorbed by the nanofibers, the $\mathrm{ZnO}$ conduction electrons are stolen, and thus conductance declines. In the case of the $\mathrm{ZnO}$ hollow nanofibers, reactive oxygen species $\left(\mathrm{O}_{2}{ }^{-}, \mathrm{O}^{2-}\right.$, and $\left.\mathrm{O}^{-}\right)$are adsorbed on the inner and outer surfaces of the hollow nanofibers at high temperatures. While at low temperatures, $\mathrm{O}_{2}{ }^{-}$ is commonly chemisorbed, at high temperatures, $\mathrm{O}^{-}$and $\mathrm{O}^{2-}$ are mainly chemisorbed, and $\mathrm{O}_{2}{ }^{-}$ vanishes immediately. Thus, the reaction kinematics could be represented as: $\mathrm{O}_{2}$ (gas) $\leftrightarrow \mathrm{O}_{2}$ (absorbed) $+\mathrm{e}^{-} \leftrightarrow \mathrm{O}_{2}^{-}+\mathrm{e}^{-} \leftrightarrow 2 \mathrm{O}^{-}$.

By exposure of the $\mathrm{ZnO}$ hollow nanofibers to ethanol, and thus its reaction with ionic oxygen species, the concentration of the oxygen species decreases, and the electron concentration, and thereby the conductance of the $\mathrm{ZnO}$ hollow nanofibers, increases. This fact can be demonstrated by the following reaction: $\mathrm{CH}_{3} \mathrm{CH}_{2} \mathrm{OH}$ (absorbed) $+6 \mathrm{O}^{-}$(absorbed) $\rightarrow 2 \mathrm{CO}_{2}+3 \mathrm{H}_{2} \mathrm{O}+6 \mathrm{e}^{-}$. The optimum ethanol sensing ability of the $\mathrm{ZnO}$ hollow nanofibers could be attributed to their 1D hollow nanofiber structure (with a large length/diameter ratio from the nanofiber structure, and a high surface/volume ratio for the inner and outer surface of the hollow structure). This interesting nanostructure facilitates fast mass transfer of ethanol molecules to and from the interaction region. In addition, it rises the rate for charge carriers to traverse the barriers induced by molecular recognition along the nanofibers.

Figure 10b shows the sensitivity of the $\mathrm{ZnO}$ hollow nanofibers when the operating temperature varies. The ethanol concentration used was $1000 \mathrm{ppm}$. The highest sensitivity $\left(R_{\mathrm{a}} / R_{\mathrm{g}}=51\right)$ was measured at the temperature of $270{ }^{\circ} \mathrm{C}$. As shown in the inset image, the rapid response and recovery time of the the $\mathrm{ZnO}$ hollow nanofiber sensors were around 3 and $5 \mathrm{~s}$, respectively. Figure 10c implies the dynamic response of the sensor when subjected to $10,20,50$, and $100 \mathrm{ppm}$ of ethanol at $270{ }^{\circ} \mathrm{C}$. The measured sensitivity values were about $2.8,3.9,7.2$, and $9.4 R_{\mathrm{a}} / R_{\mathrm{g}}$ respectively. The response and recovery time were also around 3 and $5 \mathrm{~s}$, respectively. In addition, as shown in Figure 10d reporting the dependence of the sensor sensitivity to the ethanol concentration, the sensor sensitivity increases rapidly with increasing ethanol concentrations, when they are below $2000 \mathrm{ppm}$. Beyond this concentration, the rate of the response to the ethanol concentration decreases, implying that the 
sensor becomes more or less saturated. Eventually, at 5000 ppm ethanol, the sensor sensitivity reaches a plateau and a saturation state. The inset graph, showing a linear dependence curve in the range of 10-1000 ppm, further stresses the high ethanol sensing ability of the ZnO hollow nanofibers.

In addition to ethanol, the gas sensing ability of the hollow $\mathrm{ZnO}$ nanofibers for formaldehyde [155] and acetone [44] has also been proved. As a composite, Wei et al. [121] synthesized hollow $\mathrm{SnO}_{2}-\mathrm{ZnO}$ nanofibers via single-spinneret electrospinning. These hollow nanofibers showed optimum stability and remarkable sensitivity against toluene at $190{ }^{\circ} \mathrm{C}$, thanks to their hollow structure and the $\mathrm{SnO}_{2}-\mathrm{ZnO}$ hetero-junction. By themselves, $\mathrm{SnO}_{2}$ hollow nanofibers have also been considered for magneto-optoelectronic devices, as will be introduced in the next section.

\subsection{Tin Oxide Hollow Nanofibers for Magneto-Optoelectronic Devices}

Among the metal oxides, $\mathrm{SnO}_{2}$ has shown an amazing potential as a host lattice for the dilute magnetic semiconductors (DMSs), thanks to its wide band gap, native oxygen vacancies, and high carrier density [34]. DMSs are vastly demanded in modern device applications because they offer ferromagnetism (FM) and semiconducting properties simultaneously. Possessing room temperature FM and high optical transparency, $\mathrm{SnO}_{2}$ is one of the most widely studied materials for magneto-opto-electronic devices, as well. By reducing the size of $\mathrm{SnO}_{2}$ to nanoscale, the surface area of the ensuing materials increases, which, for instance, is important to improve their magnetic properties [156]. Moreover, quantum confinement effects are extraordinary in 1D structures, resulting in the unusual but beneficial variation of their electronic properties [34]. One of the extensively applied methods for the preparation of $\mathrm{SnO}_{2}$ in $1 \mathrm{D}$ nanostructures is electrospinning [157-159]. In this regard, $\mathrm{Xia}$ et al. [37] developed hollow $\mathrm{SnO}_{2}$ nanofibers by single-spinneret electrospinning. In this study, the Kirkendall effect, as a result of the concentration gradient between Sn precursors and $\mathrm{SnO}_{2}$, was responsible for the formation of hollow $\mathrm{SnO}_{2}$ nanofibers. Moreover, the lattice and surface diffusion were found to be the driving forces for the growth of nanograins on the surface of the $\mathrm{SnO}_{2}$ nanofibers.

Doping of $\mathrm{SnO}_{2}$ confers new properties to the ensuing hollow nanofibers. For instance, Mohanapriya et al. [123] prepared $\mathrm{Mn}$-doped $\mathrm{SnO}_{2}$ hollow electrospun nanofibers. The authors observed a reasonable FM transition at $10 \mathrm{~K}$ that was attributed to the precipitated impurity phases. In another relevant work, Mohanapriya et al. [34] reported that by doping Cerium (Ce) in $\mathrm{SnO}_{2}$, the optical band gap of $\mathrm{SnO}_{2}$ hollow nanofibers declined. This confirms the direct energy transfer between f-electrons of $\mathrm{Ce}$ ions and the $\mathrm{SnO}_{2}$ conduction or valence band.

As shown in Figure 11a, the UV absorption edge seen at $\sim 250 \mathrm{~nm}$ is correlated with the photo-exciton of charges from the conduction band to the valence band. When the Ce concentration increases, the absorption edge shifts to a higher wavelength corresponding to a smaller crystallite size. This shift is caused by the charge transfer between $\mathrm{SnO}_{2}$ valence or the conduction band and the $4 \mathrm{f}$ electrons of the Ce ions. The Ce concentration-dependent red shift of the absorption edge witnesses the applicability of the Ce-doped $\mathrm{SnO}_{2}$ hollow nanofibers for narrow band-gap optoelectronic devices. The Tauc plots shown in Figure $11 \mathrm{~b}$ were employed to extract the optical band gap $\left(E_{\mathrm{g}}\right)$ for undoped and Ce-doped $\mathrm{SnO}_{2}$ hollow nanofibers through the following equation:

$$
\alpha h v=A\left(h v-E_{\mathrm{g}}\right)^{n}
$$

where $\alpha$ is the absorption coefficient, $h v$ is photon energy, $\mathrm{A}$ is constant, $n=1 / 2$ for a direct band-gap semiconductor. The $E_{\mathrm{g}}$ values are obtained via extrapolation at the linear portion of the Tauc plot at $\alpha=0$. These values for the undoped and doped $\mathrm{SnO}_{2}$ nanofibers are 3.65 and $3.4 \mathrm{eV}$, respectively. Figure 11c shows the photoluminescence (PL) spectra of the doped and undoped hollow nanofibers. In these graphs, a strong emission band is seen at $240 \mathrm{~nm}$, which is in relevance with the UV excitation. Also, the violet PL emission at $390 \mathrm{~nm}$ corresponds to an energy of $3.2 \mathrm{eV}$, which is lower than that of bulk $\mathrm{SnO}_{2}(3.6 \mathrm{eV})$. This discrepancy could be correlated to the direct electronic transition between 
the donor level and the valence band. As shown in the inset Figure, the Ce-doped $\mathrm{SnO}_{2}$ hollow fibers also exhibit an evident blue emission with an additional peak around $420 \mathrm{~nm}$ that is induced by presence of the Ce ions. The peaks at $467 \mathrm{~nm}$ are attributed to the electron transition between the new unoccupied states of $\mathrm{Ce}^{3+} 5 \mathrm{~d}$, the excited states near the conduction band of $\mathrm{SnO}_{2}$, and the $4 \mathrm{f}$ states. As demonstrated in Figure 11d,e, the undoped hollow nanofibers can offer only diamagnetism, while the doped ones (containing 3 and 6 mol. \% of Ce) show room temperature FM resulting in the FM coupling between s- and f-electrons of the $\mathrm{SnO}_{2}$ and Ce ions, respectively. When the doping level reaches 6 mol. \%, a room temperature FM property with $19 \times 10^{-5} \mathrm{emu} \cdot \mathrm{g}^{-1}$ saturation magnetization could be obtained. In general, this study implies the applicability of the Ce-doped $\mathrm{SnO}_{2}$ hollow nanofibers for magneto-optoelectronic devices.

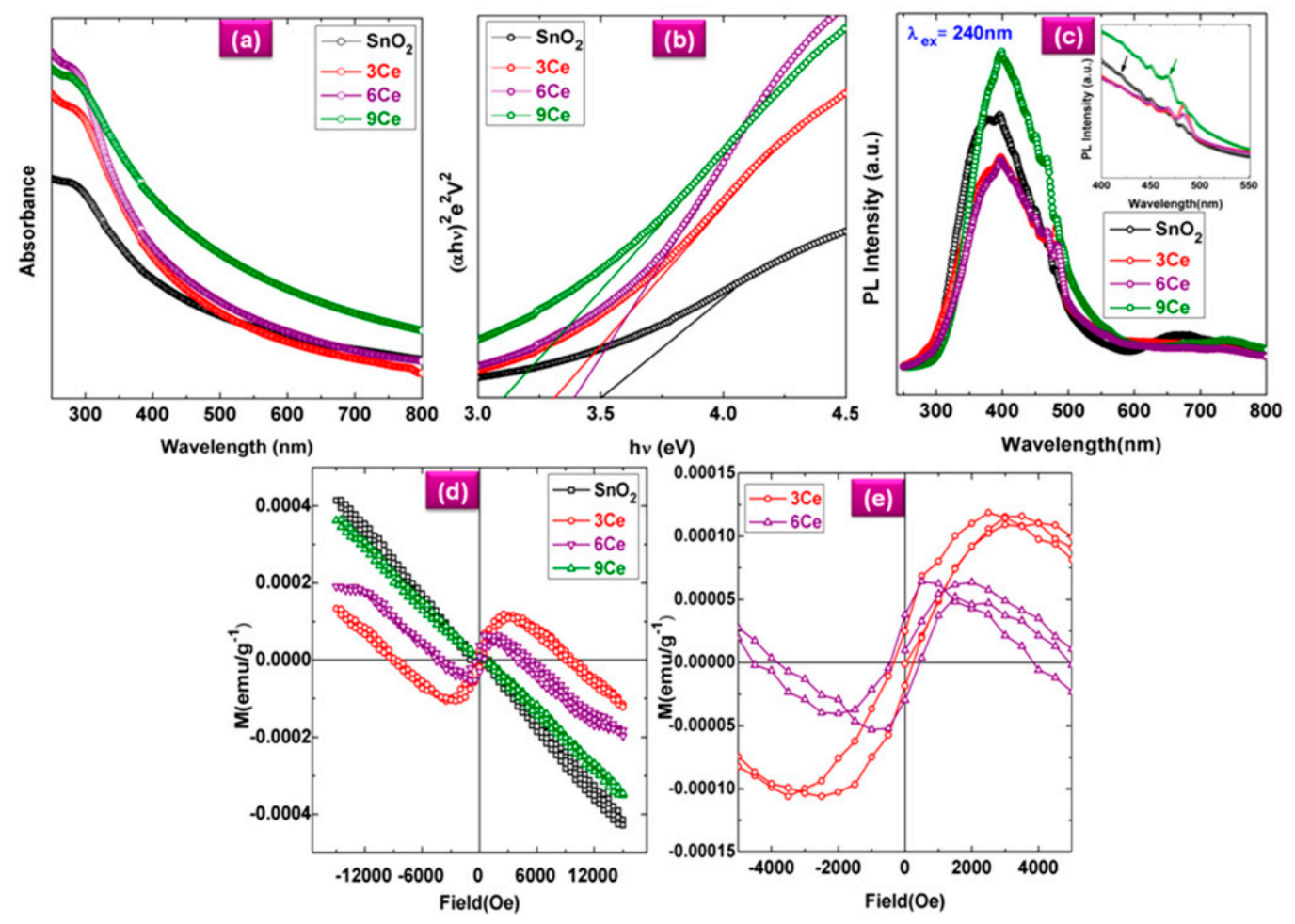

Figure 11. UV-vis absorption spectra (a) and Tauc-plot (b) of undoped and Ce-doped $\mathrm{SnO}_{2}$ hollow nanofibers, (c) photoluminescence (PL) spectra of 0, 3, 6, and 9 mol. \% Ce-doped $\mathrm{SnO}_{2}$ hollow nanofibers, (d) $\mathrm{M}-\mathrm{H}$ curves of $0,3,6$, and 9 mol. \% Ce-doped $\mathrm{SnO}_{2}$ hollow nanofibers (at $300 \mathrm{~K}$ ), and (e) extended view of the $\mathrm{M}-\mathrm{H}$ curve of 3 and 6 mol. \% Ce-doped $\mathrm{SnO}_{2}$ hollow nanofibers (Reproduced with permission from [34]. AIP Publishing LLC, 2017).

\subsection{Aluminium Oxide Hollow Nanofibers for Dye Adsorption}

Alumina $\left(\mathrm{Al}_{2} \mathrm{O}_{3}\right)$ is a bio-inert ceramic that possesses unique properties, such as high abrasion resistance, biocompatibility, and chemical inertness [160]. Hollow alumina nanofibers prepared via single-spinneret electrospinning and sintering have been studied by Peng et al. [124]. They synthesized $\gamma-\mathrm{Al}_{2} \mathrm{O}_{3}$ hollow nanofibers, as schematically shown in Figure 12a. The formation mechanism of the hollow nanofibers was based on the Kirkendall effect. The $\mathrm{N}_{2}$ adsorption-desorption isotherm was employed to extract the specific surface area of $67.17 \mathrm{~m}^{2} \cdot \mathrm{g}^{-1}$ and the mean pore size of $17.3 \mathrm{~nm}$. The authors proposed that the diameter and pore size of the hollow nanofibers can be controlled through altering the ratio of $\mathrm{Al}\left(\mathrm{NO}_{3}\right)_{3} \cdot 9 \mathrm{H}_{2} \mathrm{O}$ to PAN. The hollow nanofibers showed excellent dye adsorption efficiency for three different dyes (i.e., congo red (CR), methylene blue (MB), and acid fuchsine (AF)). 


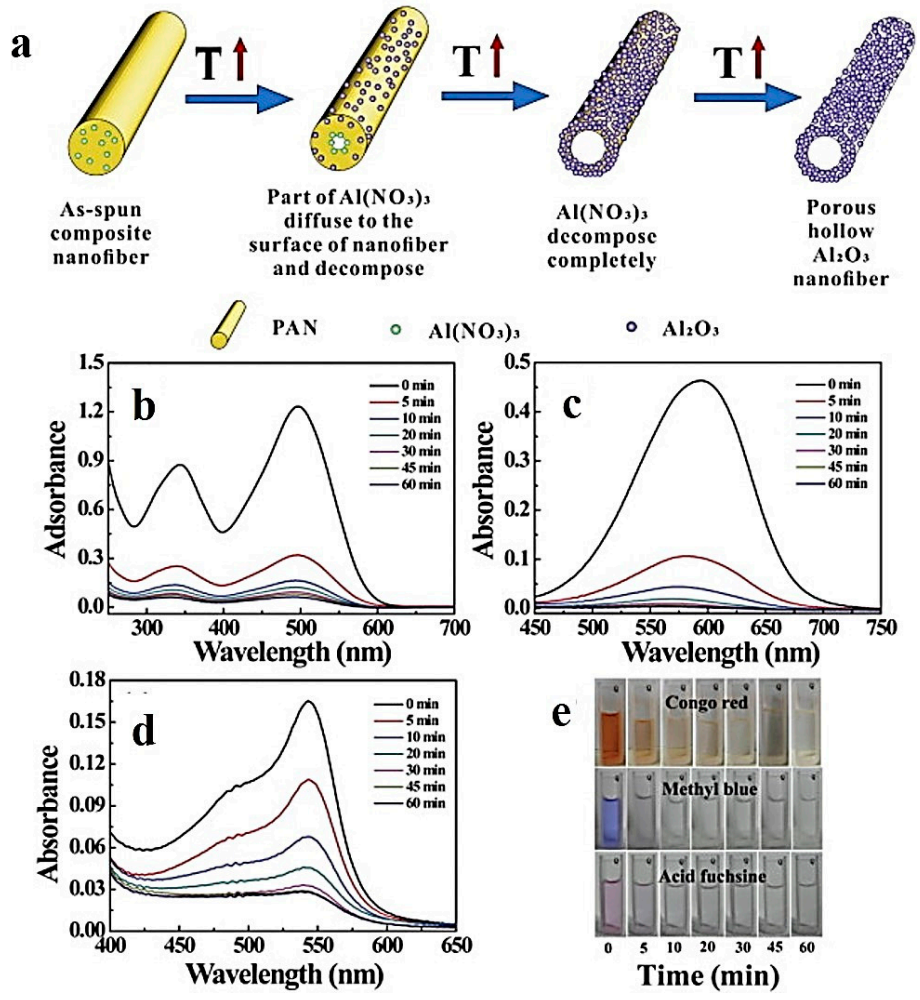

Figure 12. (a) The schematic shows different steps of the formation process of the porous hollow $\gamma-\mathrm{Al}_{2} \mathrm{O}_{3}$ nanofibers; (b-d) UV-vis spectra of congo red (CR) (b) methylene blue (MB) (c), and acid fuchsine (AF) (d) in proximity of the porous hollow $\gamma-\mathrm{Al}_{2} \mathrm{O}_{3}$ nanofibers after $0,5,10,20,30,45$, and $60 \mathrm{~min}$, respectively. (e) Camera images of the dye solutions after exposure to the adsorbent at the mentioned time intervals (Reproduced with permission from [124]. Elsevier, 2017).

UV-vis adsorption spectroscopy was implemented to determine the dye removal efficiency of the adsorbent. As seen in Figure 12b-d, the notable loss of absorbance proves the high adsorption capacity of the $\gamma-\mathrm{Al}_{2} \mathrm{O}_{3}$ nanofibers. The camera images shown in Figure 12e further confirm such an ability of the hollow nanofibers versus time.

In the UV-vis spectra, the absorbance at the corresponding wavelengths of $\mathrm{CR}, \mathrm{MB}$, and $\mathrm{AF}$ (i.e., 500, 600, and $544 \mathrm{~nm}$, respectively) was the basis to characterize the adsorption performance. As reported by the authors, with respect to CR, the adsorption process proceeds in two stages. First, the adsorption mainly occurred within a short time, so that about $75.28 \%$ of $C R$ in the solution was adsorbed in only $5 \mathrm{~min}$. Subsequently, between 5 and $60 \mathrm{~min}$, the adsorption rate decreased. After $60 \mathrm{~min}$, the CR removal efficiency was about $96.52 \%$. The adsorption process for $\mathrm{MB}$ proceeded in the same manner. The MB removal efficiency was $72.68 \%$ and $92.38 \%$ after 5 and $60 \mathrm{~min}$, respectively. On the contrary, for $\mathrm{AF}$, the primary removal after $5 \mathrm{~min}$ was not so notable, being only about $32.44 \%$. However, after $60 \mathrm{~min}$, about $91.70 \%$ of AF was removed. Thus, in the case of AF, the adsorption was done gradually.

Considering the hydroxylated surface of the $\gamma-\mathrm{Al}_{2} \mathrm{O}_{3}$ nanofibers, various interactions between $\gamma-\mathrm{Al}_{2} \mathrm{O}_{3}$ and $\mathrm{CR}$ (or MB) could be imagined: (1) likely hydrogen bonding between $-\mathrm{OH}$ on the surface of $\gamma-\mathrm{Al}_{2} \mathrm{O}_{3}$ and the $\mathrm{N}$ atom in the dye molecules, (2) electrostatic interactions resulting from excess $\mathrm{Al}^{3+}$ ions available on the surface of $\gamma-\mathrm{Al}_{2} \mathrm{O}_{3}$ nanofibers that can adsorb the dye molecules electrostatically, (3) with a lesser significance, Van Der Waals force that can play a role in the adsorption of the dye onto the surface of the porous adsorbent. 


\subsection{Vanadium Oxide Hollow Nanofibers for Gas Sensing}

Vanadium pentoxide $\left(\mathrm{V}_{2} \mathrm{O}_{5}\right)$ is a functional ceramic currently stimulating immense research interest for its employment in optical switching devices, catalysis, solar cell, sensors, etc. [10,161]. To maximize the specific surface area, many researchers have attempted to synthesize hollow nanofibers of $\mathrm{V}_{2} \mathrm{O}_{5}$. For instance, Zeng et al. [50] used emulsion electrospinning and then an annealing process to fabricate hollow $\mathrm{V}_{2} \mathrm{O}_{5}$ and $\mathrm{Au} / \mathrm{V}_{2} \mathrm{O}_{5}$ nanofibers. Both the hollow nanofibers indicated a quick response-recovery and an outstanding sensitivity against ethanol. As shown in Figure 13a, the hollow nanofibers act optimally at the operating temperatures of 200 and $220^{\circ} \mathrm{C}$, respectively. The $\mathrm{Au} / \mathrm{V}_{2} \mathrm{O}_{5}$ show an enhanced performance due to the catalytic effect of Au nanoparticles that improve surface reactions. The response/recovery time of $\mathrm{V}_{2} \mathrm{O}_{5}$ is $5 \mathrm{~s} / 5 \mathrm{~s}$, and the sensing response is $\sim 2.6 \mathrm{~s}$, while such parameters for $\mathrm{Au} / \mathrm{V}_{2} \mathrm{O}_{5}$ are 7 and $5 \mathrm{~s}$, and $\sim 2.7$, respectively, as shown in Figure 13b. Figure 13c,d, shows the dynamic ethanol sensing transients of two sensors when ethanol concentration varies from 200 to $500 \mathrm{ppm}$. The response of the sensors depends on the ethanol gas concentration, and a higher sensitivity is always seen for the composite sensor. The sensing mechanism is illustrated in Figure 13e,f, which shows that when the $\mathrm{Au} / \mathrm{V}_{2} \mathrm{O}_{5}$ sensor is exposed to air, electrons on the $\mathrm{V}_{2} \mathrm{O}_{5}$ surface move towards the Au nanoparticles mainly because of the Schottky contacts between the $\mathrm{Au}$ and the $\mathrm{V}_{2} \mathrm{O}_{5}$ nanoparticles. Concurrently, the embedded Au nanoparticles induce a spill-over effect that contributes to the catalytic activation of oxygen dissociation, enhancing the molecule-ion conversion level, and dissociating oxygen into oxygen species $\mathrm{O}^{x-}\left(\mathrm{O}_{2}{ }^{-}, \mathrm{O}^{-}\right.$and $\left.\mathrm{O}^{2-}\right)$. These species are then transported and distributed onto both the outer and inner surfaces of the nanotubes. As soon as ethanol is released, thanks to the catalytic effect, the sensing reaction between the surface $\mathrm{O}^{x-}$ and the ethanol molecules is facilitated by the Au nanoparticles. This process leads to the transfer of the trapped electrons back to $\mathrm{V}_{2} \mathrm{O}_{5}$, through the following reactions:

$$
\begin{gathered}
\mathrm{O}_{2}+2 \mathrm{~S}_{\mathrm{Au}} \rightarrow 2 \mathrm{O}-\mathrm{S}_{\mathrm{Au}} \\
\mathrm{O}-\mathrm{S}_{\mathrm{M}}+x \mathrm{e}_{\mathrm{vo}}{ }^{-}+\mathrm{S}_{\mathrm{vo}} \rightarrow \mathrm{O}^{x-} \mathrm{S}_{\mathrm{vo}}+\mathrm{S}_{\mathrm{M}} \\
\mathrm{O}^{x-} \mathrm{S}_{\mathrm{vo}}+\mathrm{C}_{2} \mathrm{H}_{5} \mathrm{OH} \rightarrow 3 \mathrm{H}_{2} \mathrm{O}+2 \mathrm{CO}_{2}+6 x \mathrm{e}_{\mathrm{vo}}{ }^{-}+\mathrm{S}_{\mathrm{vo}}
\end{gathered}
$$

where $\mathrm{S}_{\mathrm{Au}}\left(\mathrm{S}_{\mathrm{vo}}\right)$ is an adsorption site on the $\mathrm{Au}\left(\mathrm{V}_{2} \mathrm{O}_{5}\right)$, and $\mathrm{e}_{\mathrm{vo}}{ }^{-}$is an electron from $\mathrm{V}_{2} \mathrm{O}_{5}$.
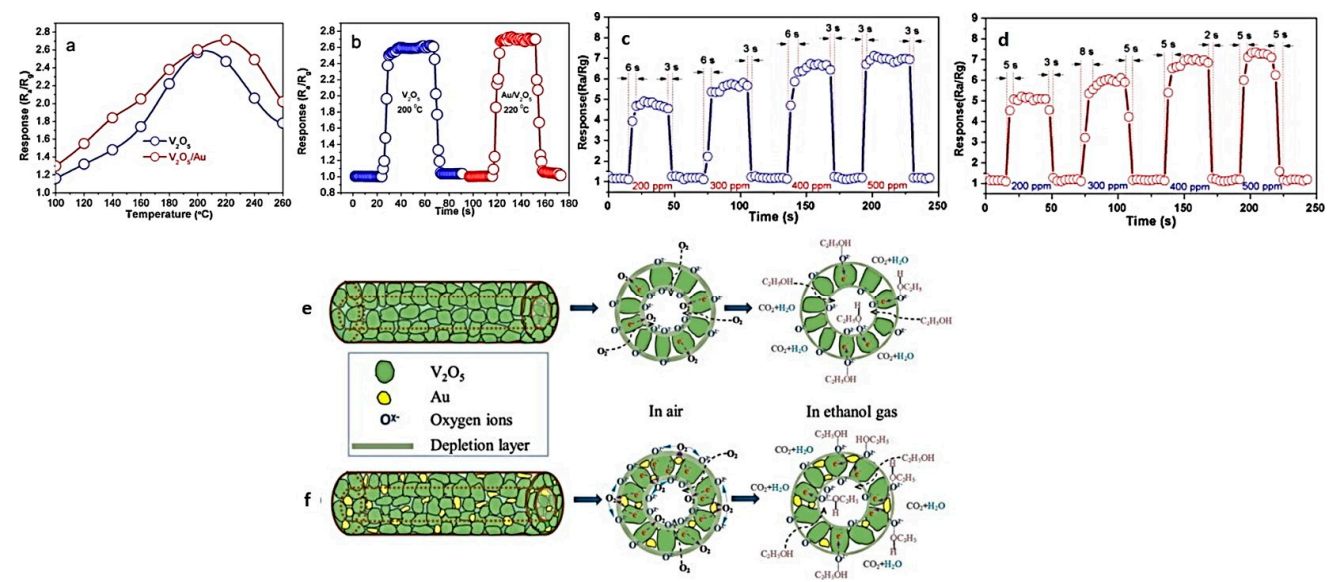

Figure 13. (a) Temperature-dependent responses of $\mathrm{V}_{2} \mathrm{O}_{5}$ and $\mathrm{Au} / \mathrm{V}_{2} \mathrm{O}_{5}$ nanotubes against ethanol; (b) response-recovery behaviors of $\mathrm{V}_{2} \mathrm{O}_{5}$ and $\mathrm{Au} / \mathrm{V}_{2} \mathrm{O}_{5}$ nanotubes exposed to ethanol. The dynamic ethanol sensing transients of (c) $\mathrm{V}_{2} \mathrm{O}_{5}$ and (d) $\mathrm{Au} / \mathrm{V}_{2} \mathrm{O}_{5}$ sensors when ethanol concentration changes from 200 to $500 \mathrm{ppm}$. The optimal operating temperature was regarded for the measurements. The schematic presentation of the sensing mechanism of (e) $\mathrm{V}_{2} \mathrm{O}_{5}$ nanotubes and (f) $\mathrm{Au} / \mathrm{V}_{2} \mathrm{O}_{5}$ nanotubes (Reproduced with permission from [50]. Elsevier, 2017). 


\section{Summary and Remarks on Future Challenges}

Hollow nanofibers are a fascinating class of nanomaterials that have gained extensive research interests for advanced applications in energy, environment remediation and biomedicine. This considerable attention stems from their size effects, surface effects, superparamagnetism, large length to diameter ratios, extensive surface area per unit mass, and small diameters. Such amazing features make them eligible for a diverse range of advanced applications in relevance with chemical sensors, photocatalysis, electromagnetic wave absorbing materials, etc.

In this review, we have focused on ceramic hollow nanofibers, and surveyed different synthesis approaches based on electrospinning, as well as their most studied types in terms of composition (i.e., metal oxide types). Among the derivative techniques of electrospinning, coaxial electrospinning is indeed the most widely employed method for the production of ceramic hollow nanofibers. With respect to the ceramic hollow nanofiber material, metal oxides have been extensively investigated for the production of hollow nanofibers for electrodes, photocatalytic adsorbents, sensors etc. For this reason, they were precisely introduced in this review, and their promising features were thoroughly discussed according to the literature. As we mentioned, metal oxide hollow nanofibers are able to develop an extensive surface area that, depending on the employed metal oxide, offers various amazing functionalities. For instance, $\mathrm{ZnO}$ and $\mathrm{V}_{2} \mathrm{O}_{5}$ hollow nanofibers provide quick response-recovery and outstanding sensitivity against various gases. As shown for $\gamma-\mathrm{Al}_{2} \mathrm{O}_{3}$, this feature enables an efficient adsorption-based removal of organics, e.g., dyes, from wastewater streams, electrostatically. In addition, hollow nanofibers of magnetic metal oxides such as $\mathrm{SnO}_{2}$, ferrites, and iron oxides induce superior magnetic properties. In addition, the quantum confinement effects are also notable in these 1D structures resulting in the unusual but beneficial variation of their electronic properties. With respect to photocatalytic metal oxides, e.g., $\mathrm{TiO}_{2}$, the photocatalytic activity is enhanced in large surface areas of the hollow nanofibers, bringing about the efficient degradation of inorganic and organic molecules. In fact, a large surface area leads to a rapid charging-discharging rate because of the small diffusion length and the high surface area.

Despite highly remarkable properties and potentials for future cutting-edge applications, there are several challenges with regard to the production of ceramic hollow nanofibers. Such difficulties must be addressed and circumvented for the sake of industrialization and upscaling. One of the greatest challenges in this regard is the lack of consistency of the core-shell morphology throughout the length of the fiber. Although what is presented in the literature about the core-shell and hollow fibers often implies a homogenous structure, this is not convincing when a much wider scope, rather than a selected TEM image, is considered. It is noteworthy that a continuous and consistent core-shell morphology necessitates a stable and steady injection of the core and the shell solution during electrospinning. However, this is not possible without any disruption to the core solution feed during the entire spinning process. Also, due to the post-treatment processes applied to remove the core, continuous and perfect hollow nanofibers are hardly made. In fact, the complete elimination of the core is challenging. The sheath layer should be strong enough to retain the hollow structure, otherwise the produced hollow nanofibers will collapse. Additionally, the limited number of suitable inner solvents and the lack of control over electrospinning parameters are other problems that can hinder the applicability of the coaxial electrospinning for some systems. Despite such challenges, the need of a uniform hollow (or core-shell) structure at a large scale depends on the envisaged application. For instance, for the sake of drug delivery, a non-continuous core-shell structure does not affect adversely the drug delivery capability. However, for other applications wherein such a structure is of utmost importance, this requirement should be investigated. As $\mathrm{Na}$ et al. [73] suggested, the capillary action of the hollow fiber could be tested to prove the continuity of the hollow fiber structure. For this reason, one end of the hollow fiber is dipped in a fluid, such as silicone oil, while the other end is left open in the air. Under such a circumstance, if the silicone oil permeates throughout the hollow fiber, the fiber is demonstrated to possess a continuous hollow core. 
The other significant challenge is related to the scalability of the technique. In general, along with the rapid progress of nanotechnology, nanofibers and their products are more demanded now than ever, but the throughput of the conventional electrospinning is not high enough to satisfy such needs. Therefore, methods able to increase the yields of the electrospun nanofibers are necessary and should be developed.

Acknowledgments: Mady Elbahri thanks the school of Chemical Engineering at Aalto University, Finland, for providing financial support for his research group.

Author Contributions: Shahin Homaeigohar and Mady Elbahri conceived the topic. Shahin Homaeigohar and Yalda Davoudpour outlined the paper and mainly wrote it. Youssef Habibi and Mady Elbahri contributed to the drafting of the manuscript.

Conflicts of Interest: The authors declare no conflict of interest.

\section{References}

1. Sun, B.; Long, Y.Z.; Zhang, H.D.; Li, M.M.; Duvail, J.L.; Jiang, X.Y.; Yin, H.L. Advances in three-dimensional nanofibrous macrostructures via electrospinning. Prog. Polym. Sci. 2014, 39, 862-890. [CrossRef]

2. Doshi, J.; Reneker, D.H. Electrospinning process and applications of electrospun fibers. J. Electrost. 1995, 35, 151-160. [CrossRef]

3. Reneker, D.H.; Chun, I. Nanometre diameter fibres of polymer, produced by electrospinning. Nanotechnology 1996, 7, 216. [CrossRef]

4. Fong, H.; Chun, I.; Reneker, D. Beaded nanofibers formed during electrospinning. Polymer 1999, 40, 4585-4592. [CrossRef]

5. Kim, J.S.; Reneker, D.H. Mechanical properties of composites using ultrafine electrospun fibers. Polym. Compos. 1999, 20, 124-131. [CrossRef]

6. Bognitzki, M.; Czado, W.; Frese, T.; Schaper, A.; Hellwig, M.; Steinhart, M.; Greiner, A.; Wendorff, J.H. Nanostructured fibers via electrospinning. Adv. Mater. 2001, 13, 70-72. [CrossRef]

7. Homaeigohar, S.; Dai, T.; Elbahri, M. Biofunctionalized nanofibrous membranes as super separators of protein and enzyme from water. J. Colloid Interface Sci. 2013, 406, 86-93. [CrossRef] [PubMed]

8. Homaeigohar, S.; Disci-Zayed, D.; Dai, T.; Elbahri, M. Biofunctionalized nanofibrous membranes mimicking carnivorous plants. Bioinspired Biomim. Nanobiomater. 2013, 2, 186-193. [CrossRef]

9. Homaeigohar, S.; Elbahri, M. Nanocomposite electrospun nanofiber membranes for environmental remediation. Materials 2014, 7, 1017-1045. [CrossRef] [PubMed]

10. Homaeigohar, S.; Zillohu, A.U.; Abdelaziz, R.; Hedayati, M.K.; Elbahri, M. A novel nanohybrid nanofibrous adsorbent for water purification from dye pollutants. Materials 2016, 9, 848. [CrossRef] [PubMed]

11. Homaeigohar, S.S.; Buhr, K.; Ebert, K. Polyethersulfone electrospun nanofibrous composite membrane for liquid filtration. J. Membr. Sci. 2010, 365, 68-77. [CrossRef]

12. Homaeigohar, S.S.; Elbahri, M. Novel compaction resistant and ductile nanocomposite nanofibrous microfiltration membranes. J. Colloid Interface Sci. 2012, 372, 6-15. [CrossRef] [PubMed]

13. Fang, X.; Reneker, D. DNA fibers by electrospinning. J. Macromol. Sci. B 1997, 36, 169-173. [CrossRef]

14. Fong, H.; Reneker, D.H. Elastomeric nanofibers of styrene-butadiene-styrene triblock copolymer. J. Polym. Sci. Polym. Phys. 1999, 37, 3488-3493. [CrossRef]

15. Beachley, V.; Wen, X. Polymer nanofibrous structures: Fabrication, biofunctionalization, and cell interactions. Prog. Polym. Sci. 2010, 35, 868-892. [CrossRef] [PubMed]

16. He, J.H.; Wan, Y.Q.; Yu, J.Y. Application of vibration technology to polymer electrospinning. Int. J. Nonlinear Sci. Numer. Simul. 2004, 5, 253-262. [CrossRef]

17. Huang, Z.-M.; Zhang, Y.-Z.; Kotaki, M.; Ramakrishna, S. A review on polymer nanofibers by electrospinning and their applications in nanocomposites. Compos. Sci. Technol. 2003, 63, 2223-2253. [CrossRef]

18. Dzenis, Y. Spinning continuous fibers for nanotechnology. Science 2004, 304, 1917-1919. [CrossRef] [PubMed]

19. Agarwal, S.; Greiner, A.; Wendorff, J.H. Electrospinning of manmade and biopolymer nanofibers-progress in techniques, materials, and applications. Adv. Funct. Mater. 2009, 19, 2863-2879. [CrossRef]

20. Zhang, Z.; Li, X.; Wang, C.; Wei, L.; Liu, Y.; Shao, C. Zno hollow nanofibers: Fabrication from facile single capillary electrospinning and applications in gas sensors. J. Phys. Chem. C 2009, 113, 19397-19403. [CrossRef] 
21. Zhang, T.; Ge, L.; Wang, X.; Gu, Z. Hollow $\mathrm{TiO}_{2}$ containing multilayer nanofibers with enhanced photocatalytic activity. Polymer 2008, 49, 2898-2902. [CrossRef]

22. Choi, K.-I.; Kim, H.-R.; Lee, J.-H. Enhanced co sensing characteristics of hierarchical and hollow $\mathrm{In}_{2} \mathrm{O}_{3}$ microspheres. Sens. Actuators B-Chem. 2009, 138, 497-503. [CrossRef]

23. Zhang, H.; Ye, F.; Liu, L.; Xu, H.; Sun, C. Synthesis of indium tin oxide nanotubes using 2-methoxyethanol as solvent via simple template method. J. Alloys Compd. 2010, 504, 171-176. [CrossRef]

24. Ab Kadir, R.; Li, Z.; Sadek, A.Z.; Abdul Rani, R.; Zoolfakar, A.S.; Field, M.R.; Ou, J.Z.; Chrimes, A.F.; Kalantar-Zadeh, K. Electrospun granular hollow $\mathrm{SnO}_{2}$ nanofibers hydrogen gas sensors operating at low temperatures. J. Phys. Chem. C 2014, 118, 3129-3139. [CrossRef]

25. Chu, Z.; Cheng, H.; Xie, W.; Sun, L. Effects of diameter and hollow structure on the microwave absorption properties of short carbon fibers. Ceram. Int. 2012, 38, 4867-4873. [CrossRef]

26. Wang, Z.; Zhao, L.; Wang, P.; Guo, L.; Yu, J. Low material density and high microwave-absorption performance of hollow strontium ferrite nanofibers prepared via coaxial electrospinning. J. Alloys Compd. 2016, 687, 541-547. [CrossRef]

27. Ma, Q.; Wang, J.; Dong, X.; Yu, W.; Liu, G.; Xu, J. Electrospinning preparation and properties of magnetic-photoluminescent bifunctional coaxial nanofibers. J. Mater. Chem. 2012, 22, 14438-14442. [CrossRef]

28. Choi, S.-H.; Ankonina, G.; Youn, D.-Y.; Oh, S.-G.; Hong, J.-M.; Rothschild, A.; Kim, I.-D. Hollow ZnO nanofibers fabricated using electrospun polymer templates and their electronic transport properties. ACS Nano 2009, 3, 2623-2631. [CrossRef] [PubMed]

29. Chang, G.; Zheng, X.; Chen, R.; Chen, X.; Chen, L.; Chen, Z. Silver nanoparticles filling in $\mathrm{TiO}_{2}$ hollow nanofibers by coaxial electrospinning. Acta Phys. Chim. Sin. 2008, 24, 1790-1797. [CrossRef]

30. Nagamine, S.; Ochi, S.; Ohshima, M. Fabrication of $\mathrm{TiO}_{2}$ hollow fibers with surface nanostructure. Mater. Res. Bull. 2011, 46, 2328-2332. [CrossRef]

31. Dong, X.; Wang, J.; Cui, Q.; Liu, G.; Yu, W. Fabrication of $\mathrm{LaNiO}_{3}$ porous hollow nanofibers via an electrospinning technique. Mod. Appl. Sci. 2008, 3, 75. [CrossRef]

32. Yao, P.-J.; Wang, J.; Qiao, Q.; Du, H.-Y. Direct fabrication of $\mathrm{La}_{0.7} \mathrm{Sr}_{0.3} \mathrm{FeO}_{3}$ nanofibers with tunable hollow structures by electrospinning and their gas sensing properties. J. Mater. Sci. 2015, 50, 1338-1349. [CrossRef]

33. $\mathrm{Gu}, \mathrm{Y}$; Jian, F. Hollow $\mathrm{LiNi}_{0.8} \mathrm{Co}_{0.1} \mathrm{Nn}_{0.1} \mathrm{O}_{2}-\mathrm{MgO}$ coaxial fibers: Sol-gel method combined with Co-electrospun preparation and electrochemical properties. J. Phys. Chem. C 2008, 112, 20176-20180. [CrossRef]

34. Mohanapriya, P.; Pradeepkumar, R.; Victor Jaya, N.; Natarajan, T. Magnetic and optical properties of electrospun hollow nanofibers of $\mathrm{SnO}_{2}$ doped with Ce-ion. Appl. Phys. Lett. 2014, 105, 022406. [CrossRef]

35. Li, D.; Dong, X.; Yu, W.; Wang, J.; Liu, G. Synthesis and upconversion luminescence properties of $\mathrm{YF}_{3}: \mathrm{Yb}^{3+} / \mathrm{Er}^{3+}$ hollow nanofibers derived from $\mathrm{Y}_{2} \mathrm{O}_{3}: \mathrm{Yb}^{3+} / \mathrm{Er}^{3+}$ hollow nanofibers. J. Nanopart. Res. 2013, 15, 1704. [CrossRef]

36. Zheng, G.; Yang, Y.; Cha, J.J.; Hong, S.S.; Cui, Y. Hollow carbon nanofiber-encapsulated sulfur cathodes for high specific capacity rechargeable lithium batteries. Nano Lett. 2011, 11, 4462-4467. [CrossRef] [PubMed]

37. Xia, X.; Dong, X.; Wei, Q.; Cai, Y.; Lu, K. Formation mechanism of porous hollow $\mathrm{SnO}_{2}$ nanofibers prepared by one-step electrospinning. Express Polym. Lett. 2012, 6, 169-176. [CrossRef]

38. Niu, H.; Lin, T. Fiber generators in needleless electrospinning. J. Nanomater. 2012, 2012, 12. [CrossRef]

39. Shadi, L.; Karimi, M.; Ramazani, S.; Entezami, A.A. Preparation of electrospun nanofibers of star-shaped polycaprolactone and its blends with polyaniline. J. Mater. Sci. 2014, 49, 4844-4854. [CrossRef]

40. Ding, Y.; Hou, H.; Zhao, Y.; Zhu, Z.; Fong, H. Electrospun polyimide nanofibers and their applications. Prog. Polym. Sci. 2016, 61, 67-103. [CrossRef]

41. Rodríguez, K.; Gatenholm, P.; Renneckar, S. Electrospinning cellulosic nanofibers for biomedical applications: Structure and in vitro biocompatibility. Cellulose 2012, 19, 1583-1598. [CrossRef]

42. Mokhena, T.; Jacobs, V.; Luyt, A. A review on electrospun bio-based polymers for water treatment. Express Polym. Lett. 2015, 9, 839-880. [CrossRef]

43. Celebioglu, A.; Uyar, T. Electrospun porous cellulose acetate fibers from volatile solvent mixture. Mater. Lett. 2011, 65, 2291-2294. [CrossRef]

44. Wei, S.; Zhou, M.; Du, W. Improved acetone sensing properties of zno hollow nanofibers by single capillary electrospinning. Sens. Actuators B Chem. 2011, 160, 753-759. [CrossRef]

45. Yu, W.; Ma, Q.; Li, X.; Dong, X.; Wang, J.; Liu, G. One-pot coaxial electrospinning fabrication and properties of magnetic-luminescent bifunctional flexible hollow nanofibers. Mater. Lett. 2014, 120, 126-129. [CrossRef] 
46. Liu, Y.; Ma, Q.; Yang, M.; Dong, X.; Yang, Y.; Wang, J.; Yu, W.; Liu, G. Flexible hollow nanofibers: Novel one-pot electrospinning construction, structure and tunable luminescence-electricity-magnetism trifunctionality. Chem. Eng. J. 2016, 284, 831-840. [CrossRef]

47. Chang, W.; Xu, F.; Mu, X.; Ji, L.; Ma, G.; Nie, J. Fabrication of nanostructured hollow $\mathrm{TiO}_{2}$ nanofibers with enhanced photocatalytic activity by coaxial electrospinning. Mater. Res. Bull. 2013, 48, 2661-2668. [CrossRef]

48. Zhao, T.; Liu, Z.; Nakata, K.; Nishimoto, S.; Murakami, T.; Zhao, Y.; Jiang, L.; Fujishima, A. Multichannel $\mathrm{TiO}_{2}$ hollow fibers with enhanced photocatalytic activity. J. Mater. Chem. 2010, 20, 5095-5099. [CrossRef]

49. Kalra, V.; Lee, J.H.; Park, J.H.; Marquez, M.; Joo, Y.L. Confined assembly of asymmetric block-copolymer nanofibers via multiaxial jet electrospinning. Small 2009, 5, 2323-2332. [CrossRef] [PubMed]

50. Zeng, W.; Chen, W.; Li, Z.; Zhang, H.; Li, T. Rapid and sensitive ethanol sensor based on hollow Au/ $\mathrm{V}_{2} \mathrm{O}_{5}$ nanotubes via emulsion-electrospinning route. Mater. Res. Bull. 2015, 65, 157-162. [CrossRef]

51. Wang, T.; Wei, J.; Shi, H.; Zhou, M.; Zhang, Y.; Chen, Q.; Zhang, Z. Preparation of electrospun Ag/ TiO 2 nanotubes with enhanced photocatalytic activity based on water/oil phase separation. Physica E 2017, 86, 103-110. [CrossRef]

52. Qi, R.; Guo, R.; Shen, M.; Cao, X.; Zhang, L.; Xu, J.; Yu, J.; Shi, X. Electrospun poly (lactic-co-glycolic acid)/halloysite nanotube composite nanofibers for drug encapsulation and sustained release. J. Mater. Chem. 2010, 20, 10622-10629. [CrossRef]

53. Zander, N.E.; Strawhecker, K.E.; Orlicki, J.A.; Rawlett, A.M.; Beebe, T.P. Coaxial electrospun poly(methyl methacrylate)-polyacrylonitrile nanofibers: Atomic force microscopy and compositional characterization. J. Phys. Chem. B 2011, 115, 12441-12447. [CrossRef] [PubMed]

54. Liu, P.; Zhu, Y.; Ma, J.; Yang, S.; Gong, J.; Xu, J. Preparation of continuous porous alumina nanofibers with hollow structure by single capillary electrospinning. Colloids Surf. A 2013, 436, 489-494. [CrossRef]

55. Hou, H.; Shang, M.; Wang, L.; Li, W.; Tang, B.; Yang, W. Efficient photocatalytic activities of $\mathrm{TiO}_{2}$ hollow fibers with mixed phases and mesoporous walls. Sci. Rep. 2015, 5, 15228. [CrossRef] [PubMed]

56. Zhang, H.; Zhao, C.; Zhao, Y.; Tang, G.; Yuan, X. Electrospinning of ultrafine core/shell fibers for biomedical applications. Sci. China Chem. 2010, 53, 1246-1254. [CrossRef]

57. Huang, J.; You, T. Electrospun nanofibers: From rational design, fabrication to electrochemical sensing applications. In Advances in Nanofibers; InTech: Rijeka, Croatia, 2013.

58. Zhou, F.-L.; Gong, R.-H.; Porat, I. Three-jet electrospinning using a flat spinneret. J. Mater. Sci. 2009, 44, 5501-5508. [CrossRef]

59. Nurwaha, D.; Han, W.; Wang, X. Investigation of a new needleless electrospinning method for the production of nanofibers. J. Eng. Fibers Fabr. 2013, 8, 42-49.

60. Forward, K.M.; Rutledge, G.C. Free surface electrospinning from a wire electrode. Chem. Eng. J. 2012, 183, 492-503. [CrossRef]

61. Tijing, L.D.; Choi, W.; Jiang, Z.; Amarjargal, A.; Park, C.-H.; Pant, H.R.; Im, I.-T.; Kim, C.S. Two-nozzle electrospinning of (MWNT/PU)/PU nanofibrous composite mat with improved mechanical and thermal properties. Curr. Appl. Phys. 2013, 13, 1247-1255. [CrossRef]

62. Chen, Z.; Chen, Z.; Zhang, A.; Hu, J.; Wang, X.; Yang, Z. Electrospun nanofibers for cancer diagnosis and therapy. Biomater. Sci. 2016, 4, 922-932. [CrossRef] [PubMed]

63. Lee, G.H.; Song, J.-C.; Yoon, K.-B. Controlled wall thickness and porosity of polymeric hollow nanofibers by coaxial electrospinning. Macromol. Res. 2010, 18, 571-576. [CrossRef]

64. Pakravan, M.; Heuzey, M.-C.; Ajji, A. Core-shell structured peo-chitosan nanofibers by coaxial electrospinning. Biomacromolecules 2012, 13, 412-421. [CrossRef] [PubMed]

65. Choi, S.-J.; Chattopadhyay, S.; Kim, J.J.; Kim, S.-J.; Tuller, H.L.; Rutledge, G.C.; Kim, I.-D. Coaxial electrospinning of $\mathrm{WO}_{3}$ nanotubes functionalized with bio-inspired pd catalysts and their superior hydrogen sensing performance. Nanoscale 2016, 8, 9159-9166. [CrossRef] [PubMed]

66. Zhang, J.; Choi, S.-W.; Kim, S.S. Micro-and nano-scale hollow $\mathrm{TiO}_{2}$ fibers by coaxial electrospinning: Preparation and gas sensing. J. Solid State Chem. 2011, 184, 3008-3013. [CrossRef]

67. Lee, B.-S.; Son, S.-B.; Park, K.-M.; Lee, G.; Oh, K.H.; Lee, S.-H.; Yu, W.-R. Effect of pores in hollow carbon nanofibers on their negative electrode properties for a lithium rechargeable battery. ACS Appl. Mater. Interfaces 2012, 4, 6702-6710. [CrossRef] [PubMed] 
68. Lee, B.-S.; Yang, H.-S.; Jung, H.; Mah, S.K.; Kwon, S.; Park, J.-H.; Lee, K.H.; Yu, W.-R.; Doo, S.-G. Facile method to improve initial reversible capacity of hollow carbon nanofiber anodes. Eur. Polym. J. 2015, 70, 392-399. [CrossRef]

69. Kaerkitcha, N.; Chuangchote, S.; Sagawa, T. Control of physical properties of carbon nanofibers obtained from coaxial electrospinning of pmma and pan with adjustable inner/outer nozzle-ends. Nanoscale Res. Lett. 2016, 11, 186. [CrossRef] [PubMed]

70. Ning, J.; Yang, M.; Yang, H.; Xu, Z. Tailoring the morphologies of PVDF nanofibers by interfacial diffusion during coaxial electrospinning. Mater. Des. 2016, 109, 264-269. [CrossRef]

71. Lee, B.-S.; Jeon, S.-Y.; Park, H.; Lee, G.; Yang, H.-S.; Yu, W.-R. New electrospinning nozzle to reduce jet instability and its application to manufacture of multi-layered nanofibers. Sci. Rep. 2013, 4, 6758. [CrossRef] [PubMed]

72. Loscertales, I.G.; Barrero, A.; Márquez, M.; Spretz, R.; Velarde-Ortiz, R.; Larsen, G. Electrically forced coaxial nanojets for one-step hollow nanofiber design. J. Am. Chem. Soc. 2004, 126, 5376-5377. [CrossRef] [PubMed]

73. Na, H.; Chen, P.; Wong, S.-C.; Hague, S.; Li, Q. Fabrication of PVDF/PVA microtubules by coaxial electrospinning. Polymer 2012, 53, 2736-2743. [CrossRef]

74. Qian, W.; Yu, D.-G.; Li, Y.; Liao, Y.-Z.; Wang, X.; Wang, L. Dual drug release electrospun core-shell nanofibers with tunable dose in the second phase. Int. J. Mol. Sci. 2014, 15, 774-786. [CrossRef] [PubMed]

75. Wei, Z.; Zhang, Q.; Peng, M.; Wang, X.; Yang, J. Preparation and drug delivery study of electrospun hollow pes ultrafine fibers with a multilayer wall. Colloid Polym. Sci. 2014, 292, 1339-1345. [CrossRef]

76. Li, X.H.; Shao, C.L.; Liu, Y.C. A simple method for controllable preparation of polymer nanotubes via a single capillary electrospinning. Langmuir 2007, 23, 10920-10923. [CrossRef] [PubMed]

77. Chen, H.; Wang, N.; Di, J.; Zhao, Y.; Song, Y.; Jiang, L. Nanowire-in-microtube structured core/shell fibers via multifluidic coaxial electrospinning. Langmuir 2010, 26, 11291-11296. [CrossRef] [PubMed]

78. Zhang, X.; Gao, X.; Jiang, L.; Qin, J. Flexible generation of gradient electrospinning nanofibers using a microfluidic assisted approach. Langmuir 2012, 28, 10026-10032. [CrossRef] [PubMed]

79. Chae, S.K.; Kang, E.; Khademhosseini, A.; Lee, S.H. Micro/nanometer-scale fiber with highly ordered structures by mimicking the spinning process of silkworm. Adv. Mater. 2013, 25, 3071-3078. [CrossRef] [PubMed]

80. Srivastava, Y.; Marquez, M.; Thorsen, T. Multijet electrospinning of conducting nanofibers from microfluidic manifolds. J. Appl. Polym. Sci. 2007, 106, 3171-3178. [CrossRef]

81. Srivastava, Y.; Loscertales, I.; Marquez, M.; Thorsen, T. Electrospinning of hollow and core/sheath nanofibers using a microfluidic manifold. Microfluid. Nanofluid. 2008, 4, 245-250. [CrossRef]

82. Nayak, R.; Padhye, R.; Kyratzis, I.L.; Truong, Y.B.; Arnold, L. Recent advances in nanofibre fabrication techniques. Text. Res. J. 2012, 82, 129-147. [CrossRef]

83. Liu, W.; Ni, C.; Chase, D.B.; Rabolt, J.F. Preparation of multilayer biodegradable nanofibers by triaxial electrospinning. ACS Macro Lett. 2013, 2, 466-468. [CrossRef]

84. Yu, D.-G.; Li, X.-Y.; Wang, X.; Yang, J.-H.; Bligh, S.; Williams, G.R. Nanofibers fabricated using triaxial electrospinning as zero order drug delivery systems. ACS Appl. Mater. Interfaces 2015, 7, 18891-18897. [CrossRef] [PubMed]

85. Han, D.; Steckl, A.J. Triaxial electrospun nanofiber membranes for controlled dual release of functional molecules. ACS Appl. Mater. Interfaces 2013, 5, 8241-8245. [CrossRef] [PubMed]

86. Khalf, A.; Singarapu, K.; Madihally, S.V. Influence of solvent characteristics in triaxial electrospun fiber formation. React. Funct. Polym. 2015, 90, 36-46. [CrossRef]

87. Zanjani, J.S.M.; Okan, B.S.; Letofsky-Papst, I.; Yildiz, M.; Menceloglu, Y.Z. Rational design and direct fabrication of multi-walled hollow electrospun fibers with controllable structure and surface properties. Eur. Polym. J. 2015, 62, 66-76. [CrossRef]

88. Zanjani, J.S.M.; Saner Okan, B.; Menceloglu, Y.Z.; Yildiz, M. Design and fabrication of multi-walled hollow nanofibers by triaxial electrospinning as reinforcing agents in nanocomposites. J. Reinf. Plast. Compos. 2015, 34, 1273-1286. [CrossRef]

89. Tang, S.; Zeng, Y.; Wang, X. Splashing needleless electrospinning of nanofibers. Polym. Eng. Sci. 2010, 50, 2252-2257. [CrossRef]

90. Zhou, F.L.; Gong, R.H.; Porat, I. Needle and needleless electrospinning for nanofibers. J. Appl. Polym. Sci. 2010, 115, 2591-2598. [CrossRef] 
91. Vonch, J.; Yarin, A.; Megaridis, C. Electrospinning: A study in the formation of nanofibers. J. Undergrad. Res. 2007, 1. [CrossRef]

92. Samanta, A.; Nandan, B.; Srivastava, R.K. Morphology of electrospun fibers derived from high internal phase emulsions. J. Colloid Interface Sci. 2016, 471, 29-36. [CrossRef] [PubMed]

93. Wei, J.; Shi, H.; Zhou, M.; Song, D.; Zhang, Y.; Pan, X.; Zhou, J.; Wang, T. Effect of oil on the morphology and photocatalysis of emulsion electrospun titanium dioxide nanomaterials. Appl. Catal. 2015, 499, 101-108. [CrossRef]

94. Elahi, F.; Lu, W.; Guoping, G.; Khan, F. Core-shell fibers for biomedical applications-A review. Bioeng. Biomed. Sci. J. 2013, 3, 1-14. [CrossRef]

95. Samanta, A.; Takkar, S.; Kulshreshtha, R.; Nandan, B.; Srivastava, R.K. Electrospun composite matrices of poly ( $\varepsilon$-caprolactone)-montmorillonite made using tenside free Pickering emulsions. Mater. Sci. Eng. 2016, 69, 685-691. [CrossRef] [PubMed]

96. Angeles, M.; Cheng, H.L.; Velankar, S.S. Emulsion electrospinning: Composite fibers from drop breakup during electrospinning. Polym. Adv. Technol. 2008, 19, 728-733. [CrossRef]

97. Choi, K.-I.; Lee, S.H.; Park, J.-Y.; Choi, D.-Y.; Hwang, C.-H.; Lee, I.-H.; Chang, M.H. Fabrication and characterization of hollow $\mathrm{TiO}_{2}$ fibers by microemulsion electrospinning for photocatalytic reactions. Mater. Lett. 2013, 112, 113-116. [CrossRef]

98. Jiang, Y.; Fang, D.; Song, G.; Nie, J.; Chen, B.; Ma, G. Fabrication of core-shell nanofibers by single capillary electrospinning combined with vapor induced phase separation. New J. Chem. 2013, 37, 2917-2924. [CrossRef]

99. Pal, J.; Singh, S.; Sharma, S.; Kulshreshtha, R.; Nandan, B.; Srivastava, R.K. Emulsion electrospun composite matrices of poly ( $\varepsilon$-caprolactone)-hydroxyapatite: Strategy for hydroxyapatite confinement and retention on fiber surface. Mater. Lett. 2016, 167, 288-296. [CrossRef]

100. Wang, W.; Zhang, L.; Tong, S.; Li, X.; Song, W. Three-dimensional network films of electrospun copper oxide nanofibers for glucose determination. Biosens. Bioelectron. 2009, 25, 708-714. [CrossRef] [PubMed]

101. Ghadiri, E.; Taghavinia, N.; Zakeeruddin, S.M.; Grätzel, M.; Moser, J.-E. Enhanced electron collection efficiency in dye-sensitized solar cells based on nanostructured $\mathrm{TiO}_{2}$ hollow fibers. Nano Lett. 2010, 10, 1632-1638. [CrossRef] [PubMed]

102. Zhang, X.; Thavasi, V.; Mhaisalkar, S.G.; Ramakrishna, S. Novel hollow mesoporous $1 \mathrm{D} \mathrm{TiO}_{2}$ nanofibers as photovoltaic and photocatalytic materials. Nanoscale 2012, 4, 1707-1716. [CrossRef] [PubMed]

103. Choi, J.-K.; Hwang, I.-S.; Kim, S.-J.; Park, J.-S.; Park, S.-S.; Jeong, U.; Kang, Y.C.; Lee, J.-H. Design of selective gas sensors using electrospun pd-doped $\mathrm{SnO}_{2}$ hollow nanofibers. Sens. Actuators B Chem. 2010, 150, 191-199. [CrossRef]

104. Cho, N.G.; Yang, D.J.; Jin, M.-J.; Kim, H.-G.; Tuller, H.L.; Kim, I.-D. Highly sensitive $\mathrm{SnO}_{2}$ hollow nanofiber-based $\mathrm{NO}_{2}$ gas sensors. Sens. Actuators B 2011, 160, 1468-1472. [CrossRef]

105. Cheng, Y.; Huang, W.; Zhang, Y.; Zhu, L.; Liu, Y.; Fan, X.; Cao, X. Preparation of $\mathrm{TiO}_{2}$ hollow nanofibers by electrospining combined with sol-gel process. CrystEngComm 2010, 12, 2256-2260. [CrossRef]

106. Peng, X.; Santulli, A.C.; Sutter, E.; Wong, S.S. Fabrication and enhanced photocatalytic activity of inorganic core-shell nanofibers produced by coaxial electrospinning. Chem. Sci. 2012, 3, 1262-1272. [CrossRef]

107. He, G.; Cai, Y.; Zhao, Y.; Wang, X.; Lai, C.; Xi, M.; Zhu, Z.; Fong, H. Electrospun anatase-phase $\mathrm{TiO}_{2}$ nanofibers with different morphological structures and specific surface areas. J. Colloid Interface Sci 2013, 398, 103-111. [CrossRef] [PubMed]

108. Tang, K.; Yu, Y.; Mu, X.; van Aken, P.A.; Maier, J. Multichannel hollow $\mathrm{TiO}_{2}$ nanofibers fabricated by single-nozzle electrospinning and their application for fast lithium storage. Electrochem. Commun. 2013, 28, 54-57. [CrossRef]

109. Lee, S.; Ha, J.; Choi, J.; Song, T.; Lee, J.W.; Paik, U. 3D cross-linked nanoweb architecture of binder-free Tio2 electrodes for lithium ion batteries. ACS Appl. Mater. Interfaces 2013, 5, 11525-11529. [CrossRef] [PubMed]

110. Jung, J.-Y.; Lee, D.; Lee, Y.-S. Cnt-embedded hollow $\mathrm{TiO}_{2}$ nanofibers with high adsorption and photocatalytic activity under uv irradiation. J. Alloys Compd. 2015, 622, 651-656. [CrossRef]

111. Adhikari, S.P.; Pant, H.R.; Mousa, H.M.; Lee, J.; Kim, H.J.; Park, C.H.; Kim, C.S. Synthesis of high porous electrospun hollow $\mathrm{TiO}_{2}$ nanofibers for bone tissue engineering application. J. Ind. Eng. Chem. 2016, 35, 75-82. [CrossRef]

112. Yang, Z.; Lu, J.; Ye, W.; Yu, C.; Chang, Y. Preparation of $\mathrm{Pt} / \mathrm{TiO}_{2}$ hollow nanofibers with highly visible light photocatalytic activity. Appl. Surf. Sci. 2017, 392, 472-480. [CrossRef] 
113. Cheng, Y.; Zou, B.; Yang, J.; Wang, C.; Liu, Y.; Fan, X.; Zhu, L.; Wang, Y.; Ma, H.; Cao, X. Fabrication of $\mathrm{CoFe}_{2} \mathrm{O}_{4}$ hollow fibers by direct annealing of the electrospun composite fibers and their magnetic properties. CrystEngComm 2011, 13, 2268-2272. [CrossRef]

114. Zhao, J.; Cheng, Y.; Yan, X.; Sun, D.; Zhu, F.; Xue, Q. Magnetic and electrochemical properties of $\mathrm{CuFe}_{2} \mathrm{O}_{4}$ hollow fibers fabricated by simple electrospinning and direct annealing. CrystEngComm 2012, 14, 5879-5885. [CrossRef]

115. Kim, K.N.; Jung, H.-R.; Lee, W.-J. Hollow cobalt ferrite-polyaniline nanofibers as magnetically separable visible-light photocatalyst for photodegradation of methyl orange. J. Photochem. Photobiol. A 2016, 321, 257-265. [CrossRef]

116. Cheng, Y.; Zou, B.; Wang, C.; Liu, Y.; Fan, X.; Zhu, L.; Wang, Y.; Ma, H.; Cao, X. Formation mechanism of $\mathrm{Fe}_{2} \mathrm{O}_{3}$ hollow fibers by direct annealing of the electrospun composite fibers and their magnetic, electrochemical properties. CrystEngComm 2011, 13, 2863-2870. [CrossRef]

117. Zhan, S.; Qiu, M.; Yang, S.; Zhu, D.; Yu, H.; Li, Y. Facile preparation of $\mathrm{MnO}_{2}$ doped $\mathrm{Fe}_{2} \mathrm{O}_{3}$ hollow nanofibers for low temperature SCR of $\mathrm{NO}$ with $\mathrm{NH}_{3}$. J. Mater. Chem. A 2014, 2, 20486-20493. [CrossRef]

118. Wei, B.-B.; Wu, Y.-B.; Yu, F.-Y.; Zhou, Y.-N. Preparation and electrochemical properties of carbon-coated $\mathrm{LiFePO}_{4}$ hollow nanofibers. Int. J. Miner. Metall. Mater. 2016, 23, 474-480. [CrossRef]

119. Xiang, H.; Long, Y.; Yu, X.; Zhang, X.; Zhao, N.; Xu, J. A novel and facile method to prepare porous hollow $\mathrm{CuO}$ and $\mathrm{Cu}$ nanofibers based on electrospinning. CrystEngComm 2011, 13, 4856-4860. [CrossRef]

120. Park, J.; Seo, S.; Kang, Y.-C.; Koh, S.-W. Tuning the oxidation states and crystallinity of copper oxide nanofibers by calcination. J. Vac. Sci. Technol. B Nanotechnol. Microelectron. 2014, 32, 04E104. [CrossRef]

121. Wei, S.; Zhang, Y.; Zhou, M. Toluene sensing properties of $\mathrm{SnO}_{2}-\mathrm{ZnO}$ hollow nanofibers fabricated from single capillary electrospinning. Solid State Commun. 2011, 151, 895-899. [CrossRef]

122. Rani, R.; Sharma, S. Preparation and characterization of $\mathrm{SnO}_{2}$ nanofibers via electrospinning. Adv. Nanopart. 2016, 5, 53. [CrossRef]

123. Mohanapriya, P.; Sathish, C.I.; Pradeepkumar, R.; Segawa, H.; Yamaura, K.; Watanabe, K.; Natarajan, T.; Jaya, N.V. Optical and magnetic studies of electrospun Mn-doped $\mathrm{SnO}_{2}$ hollow nanofiber dilute magnetic semiconductor. J. Nanosci. Nanotechnol. 2013, 13, 5391-5400. [CrossRef] [PubMed]

124. Peng, C.; Zhang, J.; Xiong, Z.; Zhao, B.; Liu, P. Fabrication of porous hollow $\gamma-\mathrm{Al}_{2} \mathrm{O}_{3}$ nanofibers by facile electrospinning and its application for water remediation. Microporous Mesoporous Mater. 2015, 215, 133-142. [CrossRef]

125. Zhao, J.; Liu, B.; Xu, S.; Yang, J.; Lu, Y. Fabrication and electrochemical properties of porous vn hollow nanofibers. J. Alloys Compd. 2015, 651, 785-792. [CrossRef]

126. Zhang, Y.; Ma, D.; Wu, J.; Zhang, Q.; Xin, Y.; Bao, N. One-step preparation of CNTs/InVO 4 hollow nanofibers by electrospinning and its photocatalytic performance under visible light. Appl. Surf. Sci. 2015, 353, 1260-1268. [CrossRef]

127. Park, H.; Jung, H.; Zhang, M.; Chang, C.H.; Ndifor-Angwafor, N.G.; Choa, Y.; Myung, N.V. Branched tellurium hollow nanofibers by galvanic displacement reaction and their sensing performance toward nitrogen dioxide. Nanoscale 2013, 5, 3058-3062. [CrossRef] [PubMed]

128. Shao, D.; Wang, J.; Dong, X.; Yu, W.; Liu, G.; Zhang, F.; Wang, L. Coaxial electrospinning fabrication and electrochemical properties of $\mathrm{LiFePO}_{4} / \mathrm{C} / \mathrm{Ag}$ composite hollow nanofibers. J. Mater. Sci. 2013, 24, 4718-4724. [CrossRef]

129. Dong, G.; Xiao, X.; Peng, M.; Ma, Z.; Ye, S.; Chen, D.; Qin, H.; Deng, G.; Liang, Q.; Qiu, J. Synthesis and optical properties of chromium-doped spinel hollow nanofibers by single-nozzle electrospinning. RSC Adv. 2012, 2, 2773-2782. [CrossRef]

130. Li, D.; Wang, J.; Dong, X.; Yu, W.; Liu, G. Fabrication and luminescence properties of $\mathrm{YF}_{3}: \mathrm{Eu}^{3+}$ hollow nanofibers via coaxial electrospinning combined with fluorination technique. J. Mater. Sci. 2013, 48, 5930-5937. [CrossRef]

131. Batool, S.S.; Imran, Z.; Rafiq, M.A.; Hasan, M.M.; Willander, M. Investigation of dielectric relaxation behavior of electrospun titanium dioxide nanofibers using temperature dependent impedance spectroscopy. Ceram. Int. 2013, 39, 1775-1783. [CrossRef]

132. Li, Z.; Zhang, H.; Zheng, W.; Wang, W.; Huang, H.; Wang, C.; MacDiarmid, A.G.; Wei, Y. Highly sensitive and stable humidity nanosensors based on licl doped $\mathrm{TiO}_{2}$ electrospun nanofibers. J. Am. Chem. Soc. 2008, 130, 5036-5037. [CrossRef] [PubMed] 
133. Jamil, H.; Batool, S.S.; Imran, Z.; Usman, M.; Rafiq, M.; Willander, M.; Hassan, M. Electrospun titanium dioxide nanofiber humidity sensors with high sensitivity. Ceram. Int. 2012, 38, 2437-2441. [CrossRef]

134. Homaeigohar, S.S.; Mahdavi, H.; Elbahri, M. Extraordinarily water permeable sol-gel formed nanocomposite nanofibrous membranes. J. Colloid Interface Sci. 2012, 366, 51-56. [CrossRef] [PubMed]

135. Tian, J.; Zhao, Z.; Kumar, A.; Boughton, R.I.; Liu, H. Recent progress in design, synthesis, and applications of one-dimensional $\mathrm{TiO}_{2}$ nanostructured surface heterostructures: A review. Chem. Soc. Rev. 2014, 43, 6920-6937. [CrossRef] [PubMed]

136. Wu, Q.; Tran, T.; Lu, W.; Wu, J. Electrospun silicon/carbon/titanium oxide composite nanofibers for lithium ion batteries. J. Power Sources 2014, 258, 39-45. [CrossRef]

137. Wang, L.; Li, J.; Wang, Y.; Zhao, L.; Jiang, Q. Adsorption capability for congo red on nanocrystalline $\mathrm{MFe}_{2} \mathrm{O}_{4}$ (M= Mn, Fe, Co, Ni) spinel ferrites. Chem. Eng. J. 2012, 181, 72-79. [CrossRef]

138. Nilmoung, S.; Kidkhunthod, P.; Pinitsoontorn, S.; Rujirawat, S.; Yimnirun, R.; Maensiri, S. Fabrication, structure, and magnetic properties of electrospun carbon/cobalt ferrite $\left(\mathrm{C} / \mathrm{COFe}_{2} \mathrm{O}_{4}\right)$ composite nanofibers. Appl. Phys. A 2015, 119, 141-154. [CrossRef]

139. Patange, S.; Shirsath, S.E.; Jadhav, S.; Hogade, V.; Kamble, S.; Jadhav, K. Elastic properties of nanocrystalline aluminum substituted nickel ferrites prepared by co-precipitation method. J. Mol. Struct. 2013, 1038, 40-44. [CrossRef]

140. Khalil, L.; Eid, C.; Bechelany, M.; Abboud, N.; Khoury, A.; Miele, P. Design of $\mathrm{CoFe}_{2} \mathrm{O}_{4} / \mathrm{Co}_{3} \mathrm{O}_{4}$ nanofibers with tunable morphology by electrospinning. Mater. Lett. 2015, 140, 27-30. [CrossRef]

141. Ammar, S.; Helfen, A.; Jouini, N.; Fievet, F.; Rosenman, I.; Villain, F.; Molinie, P.; Danot, M. Magnetic properties of ultrafine cobalt ferrite particles synthesized by hydrolysis in a polyol medium. J. Mater. Chem. 2001, 11, 186-192. [CrossRef]

142. Andrade, P.; Silva, V.; Maciel, J.; Santillan, M.; Moreno, N.; Valladares, L.D.L.S.; Bustamante, A.; Pereira, S.; Silva, M.; Aguiar, J.A. Preparation and characterization of cobalt ferrite nanoparticles coated with fucan and oleic acid. Hyperfine Interact. 2014, 224, 217-225. [CrossRef]

143. Maaz, K.; Mumtaz, A.; Hasanain, S.; Ceylan, A. Synthesis and magnetic properties of cobalt ferrite $\left(\mathrm{CoFe}_{2} \mathrm{O}_{4}\right)$ nanoparticles prepared by wet chemical route. J. Magn. Magn. Mater. 2007, 308, 289-295. [CrossRef]

144. Sangsanoh, P.; Supaphol, P. Poly (3-hydroxybutyrate)/magnetite composite nanofibers obtained via combined electrospinning and ammonia gas-enhancing in situ co-precipitation: Preparation and potential use in biomedical applications. Chiang Mai J. Sci. 2014, 41, 676-690.

145. Zhang, L.; Wu, H.B.; Lou, X.W.D. Iron-oxide-based advanced anode materials for lithium-ion batteries. Adv. Energy Mater. 2014, 4, 1300958. [CrossRef]

146. Valdiglesias, V.; Fernández-Bertólez, N.; Kiliç, G.; Costa, C.; Costa, S.; Fraga, S.; Bessa, M.J.; Pásaro, E.; Teixeira, J.P.; Laffon, B. Are iron oxide nanoparticles safe? Current knowledge and future perspectives. J. Trace Elem. Med. Biol. 2016, 38, 53-63. [CrossRef] [PubMed]

147. Binitha, G.; Soumya, M.; Madhavan, A.A.; Praveen, P.; Balakrishnan, A.; Subramanian, K.; Reddy, M.; Nair, S.V.; Nair, A.S.; Sivakumar, N. Electrospun $\alpha-\mathrm{Fe}_{2} \mathrm{O}_{3}$ nanostructures for supercapacitor applications. J. Mater. Chem. A 2013, 1, 11698-11704. [CrossRef]

148. Liu, Y.; Ma, Q.; Dong, X.; Yu, W.; Wang, J.; Liu, G. A novel strategy to directly fabricate flexible hollow nanofibers with tunable luminescence-electricity-magnetism trifunctionality using one-pot electrospinning. Phys. Chem. Chem. Phys. 2015, 17, 22977-22984. [CrossRef] [PubMed]

149. Duo, S.; Li, Y.; Zhang, H.; Liu, T.; Wu, K.; Li, Z. A facile salicylic acid assisted hydrothermal synthesis of different flower-like $\mathrm{ZnO}$ hierarchical architectures with optical and concentration-dependent photocatalytic properties. Mater. Charact. 2016, 114, 185-196. [CrossRef]

150. Li, C.; Cheng, Z.; Gao, J.; Han, Q.; Ye, M.; Zhang, J.; Huang, R.; Qu, L. Oxidation degree of graphene reflected by morphology-tailored zno growth. Carbon 2016, 107, 583-592. [CrossRef]

151. Hong, K.-S.; Kim, J.W.; Bae, J.-S.; Hong, T.E.; Jeong, E.D.; Jin, J.S.; Ha, M.G.; Kim, J.-P. Structure, chemical bonding states, and optical properties of the hetero-structured $\mathrm{ZnO} / \mathrm{CuO}$ prepared by using the hydrothermal and the electrospinning methods. Physica B 2017, 504, 103-108. [CrossRef]

152. Liu, J.; Zhu, G.; Chen, M.; Ma, X.; Yang, J. Fabrication of electrospun zno nanofiber-modified electrode for the determination of trace Cd (ii). Sens. Actuators B Chem. 2016, 234, 84-91. [CrossRef]

153. An, S.; Joshi, B.N.; Lee, M.W.; Kim, N.Y.; Yoon, S.S. Electrospun graphene-ZnO nanofiber mats for photocatalysis applications. Appl. Surf. Sci. 2014, 294, 24-28. [CrossRef] 
154. Senthil, T.; Anandhan, S. Structure-property relationship of sol-gel electrospun zno nanofibers developed for ammonia gas sensing. J. Colloid Interface Sci. 2014, 432, 285-296. [CrossRef] [PubMed]

155. Wei, S.; Zhang, Y.; Zhou, M. Formaldehyde sensing properties of ZnO-based hollow nanofibers. Sens. Rev. 2014, 34, 327-334. [CrossRef]

156. Zhao, J.-G.; Zhang, W.-Y.; Xie, E.-Q.; An, X.-Y.; Fu, J.-C.; Liu, Z.-J.; Liu, Z.-L.; Zhang, Y.-J.; Chen, Y.-F.; Zhang, C.-Y. Room-temperature ferromagnetism in $\mathrm{SnO}_{2}$ nanofibers and nanotubes prepared by electrospinning. Nano 2014, 9, 1450026. [CrossRef]

157. Asokan, K.; Park, J.Y.; Choi, S.; Chang, C.; Kim, S.S. Stabilization of the anatase phase of $\operatorname{Ti}_{1-x} \mathrm{Sn}_{x} \mathrm{O}_{2}(x<0.5)$ nanofibers. Nano Res. 2010, 3, 256-263.

158. Zhao, Y.; He, X.-L.; Li, J.-P.; Jia, J.; Gao, X.-G. Enhanced gas sensing properties of aligned porous $\mathrm{SnO}_{2}$ nanofibers. Chin. Phys. Lett. 2012, 29, 070701. [CrossRef]

159. Chandraiah, M.; Sahoo, B.; Panda, P.K. Preparation and characterization of $\mathrm{SnO}_{2}$ nanofibers by electrospinning. Trans. Indian Ceram. Soc. 2014, 73, 266-269. [CrossRef]

160. Balu, R.; Singaravelu, S.; Nagiah, N. Bioceramic nanofibres by electrospinning. Fibers 2014, 2, 221-239. [CrossRef]

161. Lala, N.L.; Jose, R.; Yusoff, M.M.; Ramakrishna, S. Continuous tubular nanofibers of vanadium pentoxide by electrospinning for energy storage devices. J. Nanopart. Res. 2012, 14, 1201. [CrossRef]

(c) 2017 by the authors. Licensee MDPI, Basel, Switzerland. This article is an open access article distributed under the terms and conditions of the Creative Commons Attribution (CC BY) license (http://creativecommons.org/licenses/by/4.0/). 